\title{
Growth and Mechanical Properties of Transition Metal Nitrides and Carbides
}

\author{
Daniel Edström
}


Growth and Mechanical Properties of Transition Metal Nitrides and Carbides

CDaniel Edström, 2016

Cover: Snapshot of TiN/TiN(001) growth simulation using after 375 ns of deposition time using flux ratio $\mathrm{N} / \mathrm{Ti}=4$ and incident $\mathrm{N}$ energy $\mathrm{E}_{\mathrm{N}}=2 \mathrm{eV}$.

Published articles are reprinted with the permission of copyright holders American Physical Society (paper 1), Elsevier (papers 2, 3, and 4), and AVS Publications (paper 5).

Printed by LiU-Tryck, Linköping, 2016

ISBN: 978-91-7685-684-0

ISSN: 0345-7524 


\section{Abstract}

The atomic-scale dynamical processes at play during film growth cannot be resolved by even the most advanced experimental methods. As such, computational methods, and chiefly classical molecular dynamics, are the only available research tools to study these processes. The investigation of key dynamical processes during thin film growth yields a deeper understanding of the film growth evolution, ultimately allowing for the optimization of experimental parameters and tailoring of film properties. This thesis details the study of fundamental surface dynamics processes, and the role played by primary diffusing species, during TiN film growth, here employed as a model system for transition metal nitrides in general. It is found that $\mathrm{Ti}$ adatoms and $\mathrm{TiN}_{2}$ admolecules are the fastest diffusing species, and the species which most rapidly descend from islands onto the growing film. Thus, they are the main contributors and players in driving the layer-bylayer growth mode. $\mathrm{TiN}_{3}$ admolecules, in contrast, are essentially stationary and thereby promote multilayer growth. Large-scale growth simulations reveal that tailoring the incident N/Ti ratio and $\mathrm{N}$ kinetic energy significantly affects the growth mode and film microstructure.

The mechanical properties of ternary transition metal nitride and carbide alloys, investigated using density functional theory, are also discussed herein, in comparison to recent experimental results. By optimizing the valence electron concentration in these compounds, the occupation of shear-compliant $d-t_{2 g}$ electronic states can be maximized. The investigation of $M^{l} M^{2} \mathrm{~N}$ alloys, where $M^{1}=\mathrm{Ti}$ or $\mathrm{V}$ and $M^{2}=\mathrm{W}$ or Mo, with different structures demonstrates that this optimization leads to enhanced ductility, and thereby toughness, in transition metal nitride alloys regardless of the degree of ordering on the metal sublattice. Estimations based on the calculation of the mechanical properties of the corresponding $M^{l} M^{2} \mathrm{C}$ transition metal carbide alloys indicate that these materials remain brittle. However, charge density analysis and calculations of stress/strain curves reveal features commonly associated with ductile materials. 


\section{Populärvetenskaplig sammanfattning}

Tunna filmer av keramiska material används regelbundet inom industrin som skyddande beläggningar på till exempel skärverktyg. För att belägga en komponent används sputtring, en teknik där det ämne som ska användas i filmen förgasas. Atomerna i gasen fäster sedan på komponenten och bildar en film allt eftersom.

Hur atomer och molekyler rör sig på ytan har stor betydelse för filmens struktur och egenskaper. Dessa rörelser är emellertid alldeles för snabba för att kunna studeras även $i$ de mest avancerade experiment. För att få insyn i förloppen på ytorna måste istället datorsimuleringar användas.

Det huvudsakliga syftet med denna avhandling är att studera denna typ av processer med hjälp av kraftfulla datorsimuleringar. Som en representant för typiska keramiska material har guldfärgad titannitrid (TiN) använts. Resultaten visar att Ti-atomer och $\mathrm{TiN}_{2}$ molekyler rör sig mycket snabbt på TiN-ytor, och hoppar också fort ner från öar av TiN. Därmed bidrar de till skapandet av en platt, jämn, film. Däremot är $\mathrm{TiN}_{3}$ molekyler $\mathrm{i}$ princip stillastående, och bidrar därmed till skapandet av en ojämn film.

Keramiska material används som skyddande filmer på grund av deras höga hårdhet. Däremot är de i allmänhet sköra, vilket leder till att filmerna spricker och tappar fästningen vid komponenterna de ska skydda. För att undvika detta är det önskvärt att utforma material som förutom att vara hårda också är böjliga.

I denna avhandling behandlas också kvantmekaniska beräkningar av de mekaniska egenskaperna i legeringar av övergångsmetallnitrider och -karbider. Resultaten visar att hårda och böjliga nitrider kan erhållas genom att optimera koncentrationen av valenselektroner, oavsett hur metallatomer är ordnade inbördes. Enkla uppskattningar baserade på mekaniska egenskaper förutspår att motsvarande legeringar av karbider är sköra. Djupare analys visar däremot egenskaper som vanligen förknippas med böjliga material. 


\section{Preface}

This thesis is the result of my doctoral studies in the Thin Film Physics division at the Department of Physics, Chemistry, and Biology (IFM), at Linköping University, between the years of 2011 and 2016.

The introductory chapters are an extension of my Licentiate thesis, A Theoretical study of mass transport processes on TiN(001) and mechanical properties of TiN-and VNbased ternaries, thesis No. 1686.

The work detailed within focuses on two main topics: simulations of processes associated with the growth of titanium nitride, employed as a model system for transition metal nitrides, and theoretical calculations of mechanical properties, specifically related to ductility, of transition metal nitride and carbide alloys. The results are published in peer-reviewed journals and are appended to this thesis.

Financial support has been provided by the Swedish Research Council, the Swedish Government Strategic Research Area Grant in Materials Science on Advanced Functional Materials, and the Knut and Alice Wallenberg Foundation.

The calculations have been performed with resources provided by the Swedish National Infrastructure for Computing at the National Supercomputer Centre in Linköping and the High Performance Computing Center North in Umeå. 


\section{Acknowledgements}

This thesis could not have been completed without the collaboration with, and support of, the following individuals:

Valeriu Chirita

Lars Hultman

Davide G. Sangiovanni.

J. E. Greene

Ivan Petrov

Additionally, I would like to thank the following people for providing company and amusing discussions.

The 9 o'clock coffee club

The Lunch Group

The Pub Group 


\section{Table of contents}

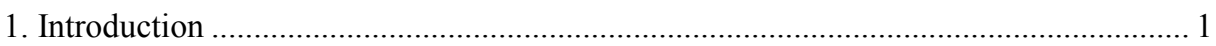

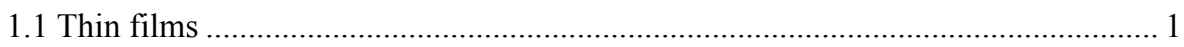

1.2. Toughness of transition-metal nitride and carbide alloys..................................... 2

1.3. Growth of titanium nitride thin films .............................................................. 4

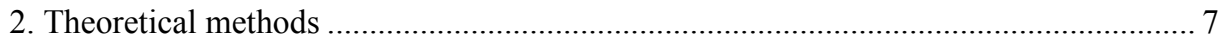

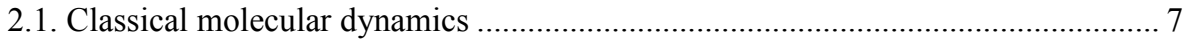

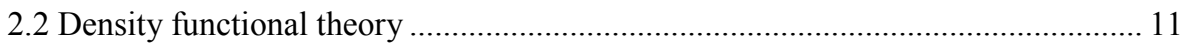

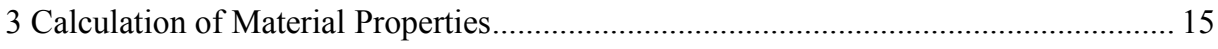

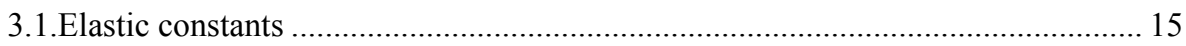

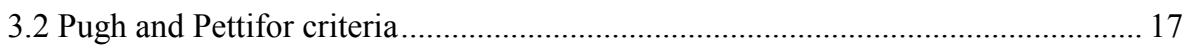

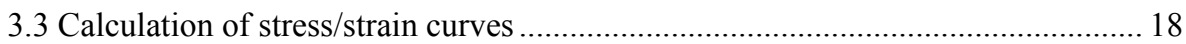

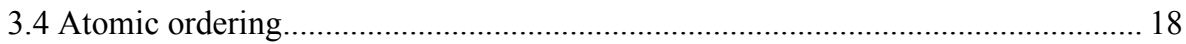

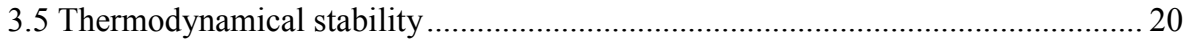

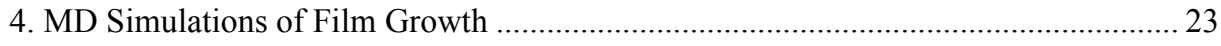

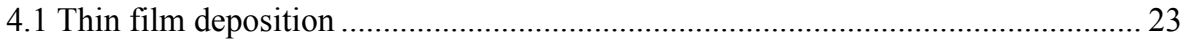

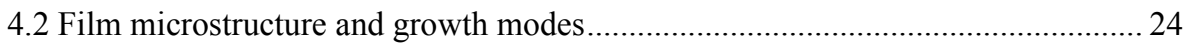

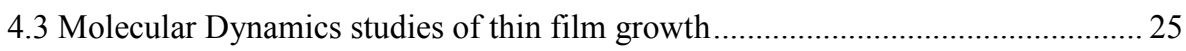

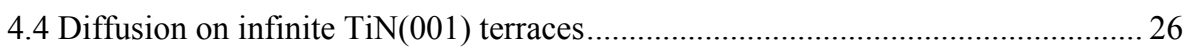

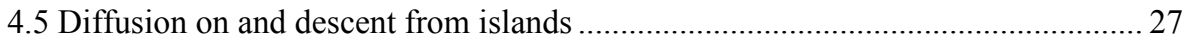

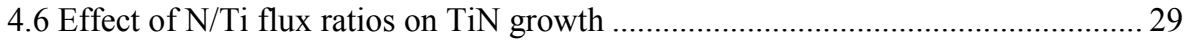

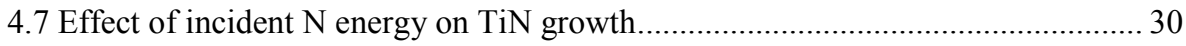

5. Mechanical properties of transition metal nitrides and carbides ................................. 33

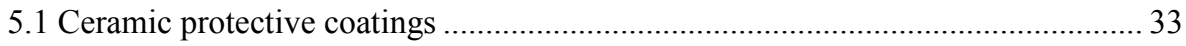

5.2 Toughness enhancement in transition metal nitride alloys..................................... 33

5.3 The effect of atomic ordering on mechanical properties of TMN alloys ............... 33

5.4 Elastic properties of ternary transition metal carbide alloys .................................... 35

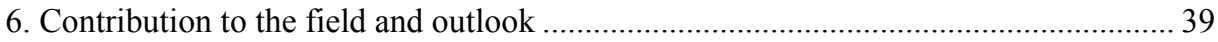

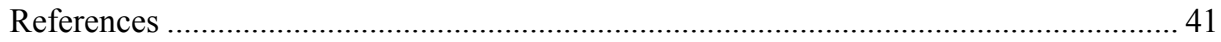

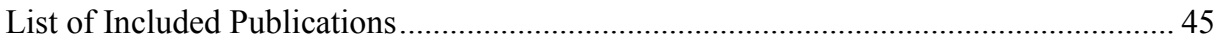

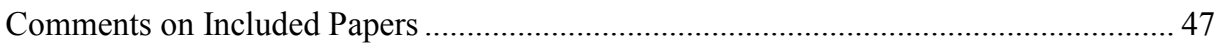




\section{Introduction}

Materials science is an interdisciplinary field which unites physics, chemistry, and engineering. Studies of materials of all forms fall within the scope of materials science: metals, semiconductors, ceramics, polymers, and biomaterials are some examples. At the heart of materials science is the relation between the structure, from atomic structure to microstructure, and the properties of the material. The material structure, and thereby the material properties, are also influenced by the processing of the material. The properties of a material are themselves intrinsically linked to the performance of a material in a given application. The understanding of the relationships between structure, properties, processing, and performance is sometimes referred to as the materials paradigm.

With the rapid evolution of computational power in the last decades, and the development of theoretical models, materials scientists now have the ability to investigate potentially interesting materials computationally before committing resources to fabrication.

\subsection{Thin films}

A thin film is any material with thickness from a single atomic layer up to several micrometers. Typically, thin films are deposited on top of a bulk material, a substrate, in order to alter the surface properties of the material. Examples of thin films include protective coatings on industrial components to prevent wear or oxidation, decorative coatings on commercial items such as watches, and even electronic components such as thin film solar cells and thin film batteries.

Transition metal nitrides and carbides are ceramic materials which share many industrially relevant properties, such as high hardness, high melting points, and high degrees of chemical inertness $[1,2]$. Consequentially, they are often employed as protective coatings on industrial cutting tools and engine components to prevent abrasion, erosion, corrosion, oxidation, and wear.

Thin films are typically deposited on a substrate by means of vapor deposition, a family of techniques divided into physical vapor deposition (PVD) and chemical vapor deposition (CVD) [3]. PVD techniques rely on vaporizing material from a target and allowing it to condense on the substrate and are carried out away from thermal equilibrium. As a consequence, the kinetics play an important role in the process, and it is possible to synthesize meta-stable phases. 
CVD, on the other hand, rely on chemical reactions between precursor gases on the substrate surface, producing the desired material [4]. Atomic layer deposition, ALD, is a class of CVD which enables the deposition of exactly one monolayer at a time until the desired film thickness is reached [5]. Since CVD does not require line-of-sight between a target and the substrate, as is the case in PVD, it is typically easier to coat complex geometries using these techniques. However, CVD is reliant on the existence of suitable precursors for the desired material.

\subsection{Toughness of transition-metal nitride and carbide alloys}

Group 4 to group 6 transition metal nitrides and carbides are materials which possess industrially relevant properties such as high hardness and wear resistance, high melting temperatures, electrical conductivity, and chemical inertness [1,2]. Due to these properties, they are commonly used in the form of thin films as diffusion barriers in microelectronics and wear-resistant coatings on industrial cutting tools and engine components. By employing these coatings, the lifetime of a component can be considerably increased, thereby reducing costs significantly.

Much effort has been put into designing ceramic coatings with improved properties, in particular increased hardness [6-8], in the last decades. Unfortunately, hard ceramics are also brittle. Consequently, the harsh conditions in, for example, industrial cutting, leads to the formation of cracks in the film surface. When the cracks propagate, the film is peeled off and the component is left unprotected. A protective coating must then not only be hard, but also ductile, meaning it must be able to dissipate mechanical energy by deforming plastically. The toughness of a material, meaning the amount of energy it can absorb before failure, is dependent on both the hardness and the ductility of the material. Figure 1.1 shows schematic stress/strain curves for a brittle and a ductile material, and their relationship to toughness. 


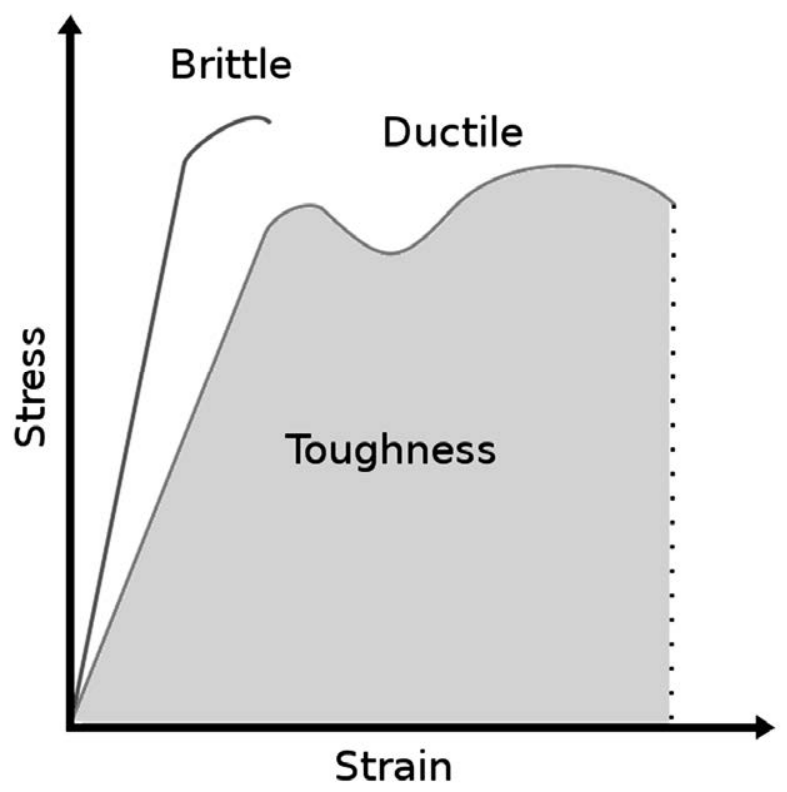

Figure 1.1: Schematic stress/strain curves for brittle and ductile materials. A brittle material may have high strength, but breaks after undergoing little plastic deformation. A ductile material deforms plastically after reaching a yield point and undergoes necking. The toughness, given as the integral of the stress/strain curve, can be significantly larger for a ductile material.

One technique for tailoring the mechanical properties of transition metal nitride and carbide alloys is tuning the valence electron concentration. For example, increased hardness was achieved by tuning the total energies of nitride and carbide phases and promoting stacking fault formation, which hinders dislocation glide [9,10]. Another example is tuning the valence electron composition so that shear-resistant metal-nitrogen bonds are fully occupied and shear-sensitive metallic states empty, and thereby maximizing plastic deformation resistance [8].

Tuning the valence electron concentration can also be used to design ductile materials. For transition metal nitride alloys, metallic $\mathrm{d}-\mathrm{t}_{2 \mathrm{~g}}$ states fill as the valence electron concentration is increased. Upon shearing of the material, this results in a layered electronic structure which promotes dislocation glide, and thereby improves ductility $[11,12]$. However, this analysis was carried out for highly ordered crystal structures, and it remains to be determined whether the same effect will be found in highly disordered systems. Paper 3 included in this thesis describes a study of the effects of configurational disorder on the metal sublattice of $M^{l} M^{2} \mathrm{~N}$ ternary alloys, where $M^{l}$ is either titanium or vanadium, and $M^{2}$ is either tungsten or molybdenum. 
Due to the similarity in properties of transition metal nitrides and carbides, it is natural to ask if the same ductility enhancement observed for transition metal nitride ternary alloys also occurs when alloying transition metal carbides. Paper 7 included in this thesis investigates, with encouraging results, $M^{l} M^{2} \mathrm{C}$ alloys, where again $M^{l}$ corresponds to titanium or vanadium and $M^{2}$ to tungsten or molybdenum.

\subsection{Growth of titanium nitride thin films}

Considering the industrial relevance of transition metal nitride thin films, there is surprisingly little knowledge of the atomistic processes at play during their growth. As properties of materials depend not only on the constituent atoms, but also significantly on their microstructure, an understanding of these processes is crucial to the ability to fine-tune deposition parameters in order to tailor the film properties.

The growth of thin films is an inherently complex process governed by both thermodynamic driving forces and kinetic mechanisms. A fundamental understanding of the mechanisms controlling the evolution of nanostructure and surface morphology necessitates detailed information about the dynamics of atomic-scale mass transport on surfaces. This includes processes such as diffusion on terraces, islands, and along edges, nucleation, coalescence, and descent from islands.

Because state-of-the-art experimental techniques, such as scanning tunneling microscopy [13-15] and low-energy electron microscopy [16-18], cannot be used to resolve the pico-second time-scale at which these processes occur it is necessary to make use of computational methods. While first-principle methods such as density functional theory (DFT) have been applied to small systems, containing up to 150 atoms, to study adatom diffusion on static transition-metal nitride surfaces [19-21], the system sizes required for studying film growth, several thousand atoms, are prohibitively large for these kinds of methods. Consequently, classical molecular dynamics (CMD) is the primary computational method for investigating atomic-scale dynamics on compound surfaces.

Titanium nitride ( $\mathrm{TiN}$ ) is one of the most well-characterized transition metal nitride coatings and thin film materials. Besides well determined bulk physical properties, a variety of techniques have been applied to study nucleation [19,22], growth [23,24], and evolution of surface morphology [25] of TiN surfaces. Due to the availability of this information, the results of simulations can be verified by comparison to experimental data. TiN interaction potentials can thus be systematically improved and used to study film growth. Due to the similarity in properties and structures of transition metal nitrides, TiN is used as a model system for most transition metal nitrides. 
Papers 1, 2, and 4 included herein present the results of studies using CMD to investigate the diffusion mechanisms of single adatoms and $\operatorname{TiNx}(\mathrm{x}=1-3)$ molecules, which have been experimentally shown to be the dominating diffusing species during TiN growth. The studies concern diffusion on $\mathrm{TiN}(001)$ terraces as well as diffusion on and descent mechanisms from square TiN/TiN(001) islands. Further studies are carried out in papers 5 and 6, in which large-scale simulations of TiN film deposition is carried out, and the effects of incident $\mathrm{N} / \mathrm{Ti}$ ratio and $\mathrm{N}$ energy are investigated. Experimentally, these parameters correspond to $\mathrm{N}_{2}$ partial pressure, as a means to control supersaturation, and substrate bias, which accelerates $\mathrm{N}^{+}$and $\mathrm{N}_{2}{ }^{+}$ions to the growing film. 


\section{Theoretical methods}

The work presented within this thesis relies on two computational methods: Classical molecular dynamics (CMD) using the modified embedded atom method (MEAM) interaction potential, and density functional theory (DFT). CMD is computationally efficient and can be used to efficiently simulate large systems, up to $10^{5}$ atoms, for timescales ranging up to several microseconds. For this reason, it is ideal for studies of film growth and related dynamical processes. For CMD to be applicable, an accurate interaction potential must be available for the studied system. Interaction potentials within the MEAM framework for TiN have been developed in recent years. However, there exists no such potentials for the ternary nitride and carbide alloys studied herein.

DFT, on the other hand, is a first-principles method firmly based on a quantum mechanical description. Although advancements in both algorithms and hardware are being made constantly, it is much more computationally expensive than CMD and is thus realistically limited to system sizes of a few hundred atoms and time-scales of, at best, a nanosecond. In the work presented herein, DFT is applied for the calculation of static properties.

\subsection{Classical molecular dynamics}

Classical molecular dynamics (CMD) is a technique based on treating atoms as point particles obeying Newton's equations of motion. The atoms interact with each other according to a semi-empirical potential, optimized for the studied system, which yields the forces that are needed to integrate the equations of motion. In this work, CMD has been performed using the Large-scale Atomic/Molecular Massively Parallel Simulator (LAMMPS) software, distributed by Sandi National Laboratories [26], which employs the velocity Verlet algorithm for the integration of the equations of motion,

$$
\begin{gathered}
\vec{r}(t+\Delta t)=\vec{r}(t)+\vec{v}(t) \Delta t+\frac{\vec{f}(t)}{2 m} \Delta t^{2} \\
\vec{v}(t+\Delta t)=\vec{v}(t)+\frac{\vec{f}(t+\Delta t)+\vec{f}(t)}{2 m} \Delta t .
\end{gathered}
$$

Here, the vectors $\vec{r}, \vec{v}$, and $\vec{f}$ are the position, velocity, and force, respectively, and the scalar $\Delta t$ is the time step. In order to ensure accurate results, the time step must be sufficiently short to capture the dynamics of the system. For the work presented here, a time step of 1 fs has been used. When initializing the simulation, the initial positions of the 
atoms are set in accordance with the structure of the material and the initial velocities are sampled from a Maxwell-Boltzmann distribution at the set temperature. The CMD scheme is summarized in Figure 2.1.

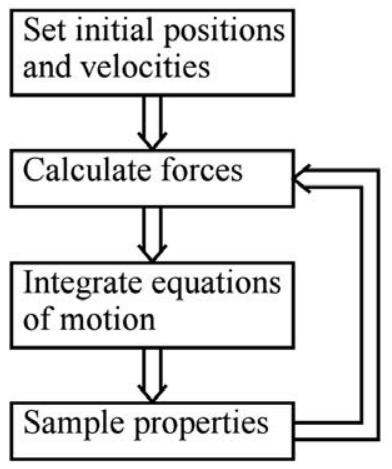

Figure 2.1: The Molecular Dynamics loop. First, initial positions and velocities of all atoms are set to correspond to the structure of the material and given temperature. Then, for each timestep of the simulation, the forces acting on all atoms are calculated from the inter-atomic potential and the equations of motion are integrated and any properties of interest are sampled.

It is also necessary to define in which statistical ensemble the simulation is performed. A typical choice is the micro-canonical, or NVE, ensemble, in which the number of particles $N$, volume $V$, and energy $E$, are conserved throughout the simulation $[27,28]$. This ensemble is used throughout the work presented here, with an additional constraint that particle velocities are rescaled at regular intervals to keep the system at the desired temperature. This is not to be confused with a formal sampling of the canonical, or NVT, ensemble, in which the system temperature, $T$, is kept constant instead of the energy. Sampling the true canonical ensemble can be achieved using more sophisticated methods such as the Andersen or Nosé-Hoover thermostats. In the Andersen thermostat, atoms are randomly subjected to elastic collisions with a heat bath maintained at the desired temperature, whereas the Nosé-Hoover thermostat enables the deterministic sampling of the canonical ensemble by introducing additional coordinates and velocities and rewriting the equations of motion, a so called extended Hamiltonian scheme [27,28].

It should also be mentioned that other ensembles than the micro-canonical and canonical ensembles can be studied using CMD as well. The isothermal-isobaric, or NPT, ensemble, in which the volume of the system is allowed to change under constant pressure, 
and the grand canonical ensemble, where the particles number is free to fluctuate can also be described using extended Hamiltonian schemes [28,29].

\subsubsection{Modified embedded atom method}

The behavior of the simulated system is determined by the choice of interatomic potential. Over the years, many different interaction potentials of varying forms and degrees of complexity have been developed. The perhaps simplest potential is the LennardJones potential, which is a pair potential that depends only on the interatomic distances. The Lennard-Jones potential has two tunable parameters: the depth and position of the potential well. Many systems require significantly more complex potentials that, in addition to interatomic distances, also take into account bond angles and screening effects.

The potential employed in the work presented here is the Modified Embedded Atom Method (MEAM) [30]. This potential is dependent on interatomic distances, bond angles, and screening effects, and has been shown to reproduce properties of several fcc, bcc, and hep metals. The potential has also been extended to binary and ternary systems, thereby enabling the simulation of transition metal nitride compounds and alloys, as in this work. In MEAM, the system energy is given as

$$
E=\sum_{i}\left[F_{i}\left(\bar{\rho}_{i}\right)+\frac{1}{2} \sum_{j \neq i} S_{i j} \varphi_{i j}\left(r_{i j}\right)\right],
$$

where $F_{i}\left(\bar{\rho}_{i}\right)$ is the energy required to embed atom $i$ within the background electron density $\bar{\rho}_{i}, \varphi_{i j}\left(r_{i j}\right)$ is the pair interaction between atoms $i$ and $j$ at distance $r_{i j}$, and $S_{i j}$ describes the screening between atoms $i$ and $j$. Through the embedding function, many-body interactions are taken into account, which is crucial when describing metals.

As suggested by the name, MEAM is a modification of the Embedded Atom Method (EAM) [31], which employs the same functional form. The key difference is how the background electron density, $\bar{\rho}_{i}$, is treated. In the original EAM, the background electron density is computed as a sum of spherically averaged atomic electron densities. Due to the spherical averaging, it is impossible to describe any form of directional bonding. In MEAM, the background density is augmented with angular dependent terms, thereby allowing for simulation of materials with covalent bonding [30].

In a many-body system, the interaction between atoms may be partially, or even completely, screened by atoms located between them. In MEAM, this is described by the screening function $S_{i j}$ [32], which is itself a product of the screening caused by all individual atoms $k$. Expressed mathematically, 


$$
S_{i j}=\prod_{k \neq i, j} S_{i j k} .
$$

The factors $S_{i j k}$ are determined via a geometrical construction, an ellipse passing through atoms $i, j$, and $k$, with the $x$-axis defined by the vector from atom $i$ to atom $j$.

$$
x^{2}+\frac{y^{2}}{C}=\left(\frac{R_{i j}}{2}\right)^{2},
$$

where $R_{i j}$ is the distance between atoms $i$ and $j$. The degree of screening is determined by the MEAM parameters $C_{\min }$ and $C_{\max }$. If f $C \leq C_{\min }, S_{i j k}=0$ and if $C \geq C_{\max }, S_{i j k}=$ 1 corresponding to total and no screening, respectively. For $C_{\min } \leq C \leq C_{\max }, S_{i j k}$ varies smoothly from 0 to 1 . The construction is illustrated in Figure 2.2.

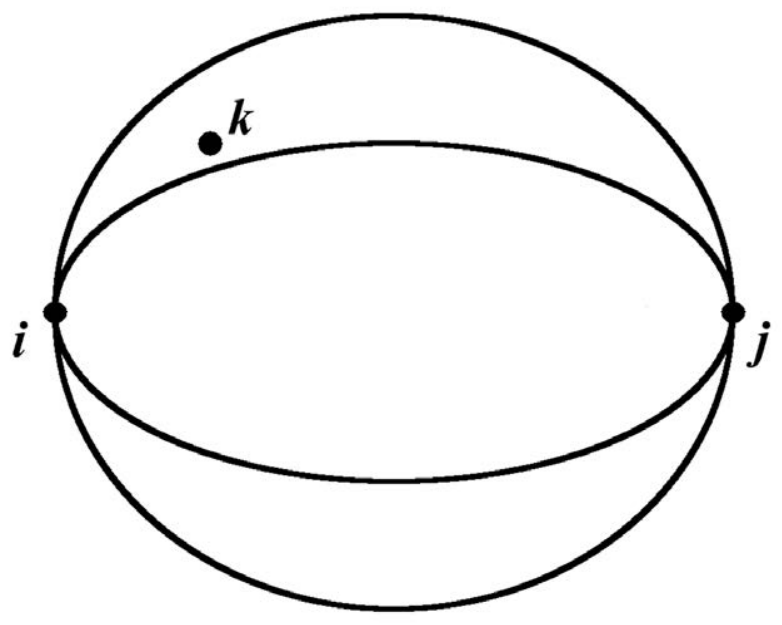

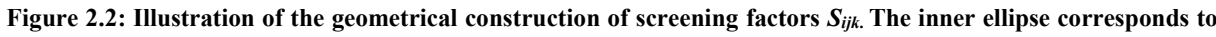
the MEAM parameter $\mathrm{C}_{\min }$, and the outer to $\mathrm{C}_{\max }$. In the example illustrated here, the interaction between atoms $i$ and $j$ is partially screened by atom $k$.

The parametrization of MEAM employed in this work to study TiN reproduces bulk and surface properties to a very high degree. Additionally, it yields adatom formation energies, diffusion barriers, Ehrlich-Schwoebel barriers consistent with experimental results, quantities which are of utmost importance when modelling processes related to film growth.

\subsubsection{Nudged elastic band}

The nudged elastic band (NEB) $[33,34]$ method is a technique for finding the minimum energy path between two states, for example a diffusion path between two adsorption sites. First, a number of replicas, or images, of the system is prepared along an 
initial guessed path between the initial and final state. A common initial path is a straight line with equidistant images. By minimizing the energy of each image, the minimum energy path can be found. To prevent the images from simply converging to the minimum energy state of the system, artificial spring forces between the images are introduced. Once the minimum energy path has been found, the image with highest energy is allowed to climb the potential landscape along the band, thereby finding the saddle point along the path. In this way, the energy barrier between the initial and final states is found.

In the studies of diffusion presented in this thesis, the NEB method has been used to determine energy barriers for descent of Ti and $\mathrm{N}$ adatoms from $\mathrm{TiN}(001)$ islands, via direct hopping as well as via push-out/exchange mechanisms. It has also been applied to determine energy barriers for adatom diffusion along island edges and corner rounding.

\subsection{Density functional theory}

In quantum mechanics, any system can be described with a many-particle wave function, which can be found by solving the Schrödinger equation. For a system of $N$ particles, the Schrödinger equation involves $3 N$ degrees of freedom, 3 for each particle. As such, the direct of solution of this equation becomes intractable for larger systems. The application of density functional theory (DFT) allows for the circumvention of this problem by rephrasing the problem in terms of the electron density $n(\vec{r})$, which depends only on three variables: the spatial coordinates. In this work, DFT has been used to calculate thermodynamical phase stability, elastic constants, and stress/strain curves.

\subsubsection{The Hohenberg-Kohn theorems}

The modern DFT can be said to originate in 1964, with the publication of the works of Hohenberg and Kohn [35] and what is now known as the Hohenberg-Kohn theorems. The theorems state the following:

Theorem 1: For any system of interacting particles in an external potential $V_{\text {ext }}(\vec{r})$, the potential $V_{\text {ext }}(\vec{r})$ is determined uniquely, up to a constant, by the ground state particle density $n_{0}(\vec{r})$.

Theorem 2: A universal functional for the energy $E[n]$ in terms of the density $\mathrm{n}(\vec{r})$ can be defined, valid for any external potential $V_{\text {ext }}(\vec{r})$. For any particular $V_{\text {ext }}(\vec{r})$, the exact ground state energy of the system is the global minimum value of this functional, and the density $n(\mathbf{r})$ that minimizes the functional is the exact ground state density $n_{0}(\vec{r})$. 
The consequence of the theorem 1 is that all properties of the system can be determined from the ground state electron density, and that of theorem 2 that knowledge of the functional $E[n]$ is enough to find the ground state density.

\subsubsection{The Kohn-Sham scheme}

In the Kohn-Sham [36] scheme the system of interest is mapped onto a system of non-interacting particles with the same ground state electron density as the real system of interacting particles. This is achieved by replacing the external potential with an effective potential $V_{\text {eff }}$ given as

$$
V_{e f f}(\vec{r})=V_{H}(\vec{r})+V_{x c}(\vec{r})+V_{\text {ext }}(\vec{r}),
$$

where $V_{H}(\vec{r})$ is the Hartree potential, $V_{x c}(\vec{r})$ the exchange-correlation potential and $V_{\text {ext }}(\vec{r})$ the ionic potential. Since the particles in this system are non-interacting, they can be described by single particle wave functions found by solving the single particle KohnSham equations

$$
\left(-\frac{1}{2} \nabla^{2}+v_{e f f}(\vec{r})\right) \varphi_{i}(\vec{r})=\varepsilon_{i} \varphi_{i}(\vec{r}),
$$

where $\varepsilon_{i}$ is the eigenvalue to the non-interacting single particle wavefunction. The electron density is then obtained by summation over all single particle orbitals

$$
n(\vec{r})=\sum_{i=1}^{N}\left|\varphi_{i}(\vec{r})\right|^{2},
$$

and the Kohn-Sham energy via the expression

$$
E_{K S}[n]=T_{S}[n]+\int d \vec{r} V_{e x t}(\vec{r}) n(\vec{r})+E_{H}[n]+E_{I I}[n]+E_{x c}[n],
$$

where the terms on the right-hand side are, from left to right, the kinetic energy, the energy due to the ionic potential, the Hartree energy, the energy due to ionic interaction, and the exchange-correlation energy. It is important to note that while the Kohn-Sham energy given here is formally exact, no exact form for the exchange-correlation energy is known, and approximations must thus be made. Common approximation for the exchangecorrelation energy include the local density approximation (LDA) [36], which take the local exchange-correlation energy density to be the same as that in a uniform electron gas of that density, and variants of the generalized gradient approximation (GGA) [37,38], in which the exchange-correlation energy is a function of the local density as well as the gradient of the density. The DFT calculations pertaining to the work presented herein have been carried out using the Vienna ab-initio simulation package (VASP) [39], GGA for 
exchange and correlation energy, and the projector augmented wave (PAW) [40] method to describe electron-ion interactions. 


\section{Calculation of Material Properties}

\subsection{Elastic constants}

The elastic properties of a material describe the relation between the strain $e$ and the stress $\sigma$, described here following the presentations of Kittel [41], and Aschroft and Mermin [42]. For an arbitrary displacement in a solid given by

$$
\vec{R}(\vec{r})=u(\vec{r}) \hat{x}+v(\vec{r}) \hat{y}+w(\vec{r}) \hat{z},
$$

the strain components are defined as

$$
\begin{gathered}
e_{1}=e_{x x}=\frac{\partial u}{\partial x}, e_{2}=e_{y y}=\frac{\partial v}{\partial y}, e_{3}=e_{z z}=\frac{\partial w}{\partial z} \\
e_{4}=e_{y z}=\frac{\partial v}{\partial z}+\frac{\partial w}{\partial y}, e_{5}=e_{z x}=\frac{\partial w}{\partial x}+\frac{\partial u}{\partial z} \\
e_{6}=e_{x y}=\frac{\partial u}{\partial y}+\frac{\partial v}{\partial x}
\end{gathered}
$$

Hooke's law, which is valid for sufficiently small deformations, states that the stress is directly proportional to the strain, and vice versa. This can be expressed with a $6 \times 6$ matrix:

$$
\left(\begin{array}{l}
\sigma_{1} \\
\sigma_{2} \\
\sigma_{3} \\
\sigma_{4} \\
\sigma_{5} \\
\sigma_{6}
\end{array}\right)=\left(\begin{array}{llllll}
C_{11} & C_{12} & C_{13} & C_{14} & C_{15} & C_{16} \\
C_{21} & C_{22} & C_{23} & C_{24} & C_{25} & C_{26} \\
C_{31} & C_{32} & C_{33} & C_{34} & C_{35} & C_{36} \\
C_{41} & C_{42} & C_{43} & C_{44} & C_{45} & C_{46} \\
C_{51} & C_{52} & C_{53} & C_{54} & C_{55} & C_{56} \\
C_{61} & C_{62} & C_{63} & C_{64} & C_{65} & C_{66}
\end{array}\right)\left(\begin{array}{l}
e_{1} \\
e_{2} \\
e_{3} \\
e_{4} \\
e_{5} \\
e_{6}
\end{array}\right)
$$

The coefficients $C_{i j}$ are known as the elastic stiffness constants, or moduli of elasticity. They are symmetrical, i. e. $C_{i j}=C_{j i}$, resulting in 21 independent constants. For many systems, the number of independent constants is reduced further by the symmetry requirements of the crystal structure. For a cubic crystal structure, like the ones studied here, only three independent constants need to be considered.

$$
\left(\begin{array}{l}
\sigma_{1} \\
\sigma_{2} \\
\sigma_{3} \\
\sigma_{4} \\
\sigma_{5} \\
\sigma_{6}
\end{array}\right)=\left(\begin{array}{cccccc}
C_{11} & C_{12} & C_{12} & 0 & 0 & 0 \\
C_{12} & C_{11} & C_{12} & 0 & 0 & 0 \\
C_{12} & C_{12} & C_{11} & 0 & 0 & 0 \\
0 & 0 & 0 & C_{44} & 0 & 0 \\
0 & 0 & 0 & 0 & C_{44} & 0 \\
0 & 0 & 0 & 0 & 0 & C_{44}
\end{array}\right)\left(\begin{array}{l}
e_{1} \\
e_{2} \\
e_{3} \\
e_{4} \\
e_{5} \\
e_{6}
\end{array}\right)
$$

Knowledge of the elastic moduli yields the elastic energy density as a function of strain, 


$$
U=\frac{1}{2} \sum_{i, j} C_{i j} e_{i} e_{j}
$$

Relation 3.5 provides a path to computationally determine the elastic constants. By applying specifically chosen distortions to the system, relations only involving one or a few elastic constants and the square of the distortion are derived.

Thus, applying a tetragonal distortion, given by the matrix

$$
\varepsilon=\left(\begin{array}{ccc}
-\delta / 2 & 0 & 0 \\
0 & -\delta / 2 & 0 \\
0 & 0 & \delta
\end{array}\right)
$$

yields a strain energy given by

$$
U_{\text {tetr }}=\frac{3}{4}\left(C_{11}-C_{12}\right) \delta^{2}
$$

whereas a trigonal distortion

$$
\varepsilon=\left(\begin{array}{lll}
0 & \delta & 0 \\
\delta & 0 & 0 \\
0 & 0 & 0
\end{array}\right)
$$

results in the energy

$$
U_{\text {trig }}=2 C_{44} \delta^{2} \text {. }
$$

In addition, for a uniform deformation, the bulk modulus, $\mathrm{B}$, is defined according to

$$
U_{\text {unif }}=\frac{1}{2} B \delta^{2} \text {. }
$$

By varying the magnitude of the distortion over a small range, $|\delta|<0.005, B, C_{44}$, and $C_{11}-C_{12}$ can be obtained directly from energy calculations using DFT. The bulk modulus is related to the elastic constants according to

$$
B=\frac{C_{11}+2 C_{12}}{3},
$$

which allows for $C_{11}$ and $C_{12}$ to be found directly from the calculated vales of $B$ and the difference $C_{11}-C_{12}$.

For alloys, even if they crystallize in a cubic structure, due to atomic disorder they might not obey the symmetry relations of cubic systems, and may require 21 elastic constants. However, it is possibly to project the elastic constants onto a system which does obey the cubic symmetry relations and calculate the projected elastic constants instead $[43,44]$. The projected elastic constants are found according to

$$
\begin{aligned}
& \bar{C}_{11}=\frac{C_{11}+C_{22}+C_{33}}{3}, \\
& \bar{C}_{12}=\frac{C_{12}+C_{23}+C_{13}}{3}, \\
& \bar{C}_{44}=\frac{C_{44}+C_{55}+C_{66}}{3},
\end{aligned}
$$




\subsection{Pugh and Pettifor criteria}

Whether a material is ductile or brittle can be estimated with knowledge of its elastic constants. Two empirical criteria for ductility, which reflect the nature of the bonding at the atomic level, have been developed. The Pettifor criterion is based on the Cauchy pressure, defined as the difference $C_{12}-C_{44}$. A negative Cauchy pressure corresponds to a brittle material, whereas positive Cauchy pressures are associated with ductility. This stems from metallic bonding being represented by positive Cauchy pressures [45].

The Pugh criterion, on the other hand, is related to the ratio between shear and bulk modulus, $G / B$ [46]. Brittleness is associated with $\mathrm{G} / \mathrm{B}<0.5$, and ductility with $\mathrm{G} / \mathrm{B}>0.5$. While the original work considered only malleability in pure metals, the criterion is today employed for compounds as well.

By employing these two criteria in tandem, the brittle-ductile nature of materials can be qualitatively predicted. It is common to illustrate the criteria graphically in a brittle/ductile map. An example is shown in figure 3.1, where TiN (brittle) and TiMoN (ductile) have been included.

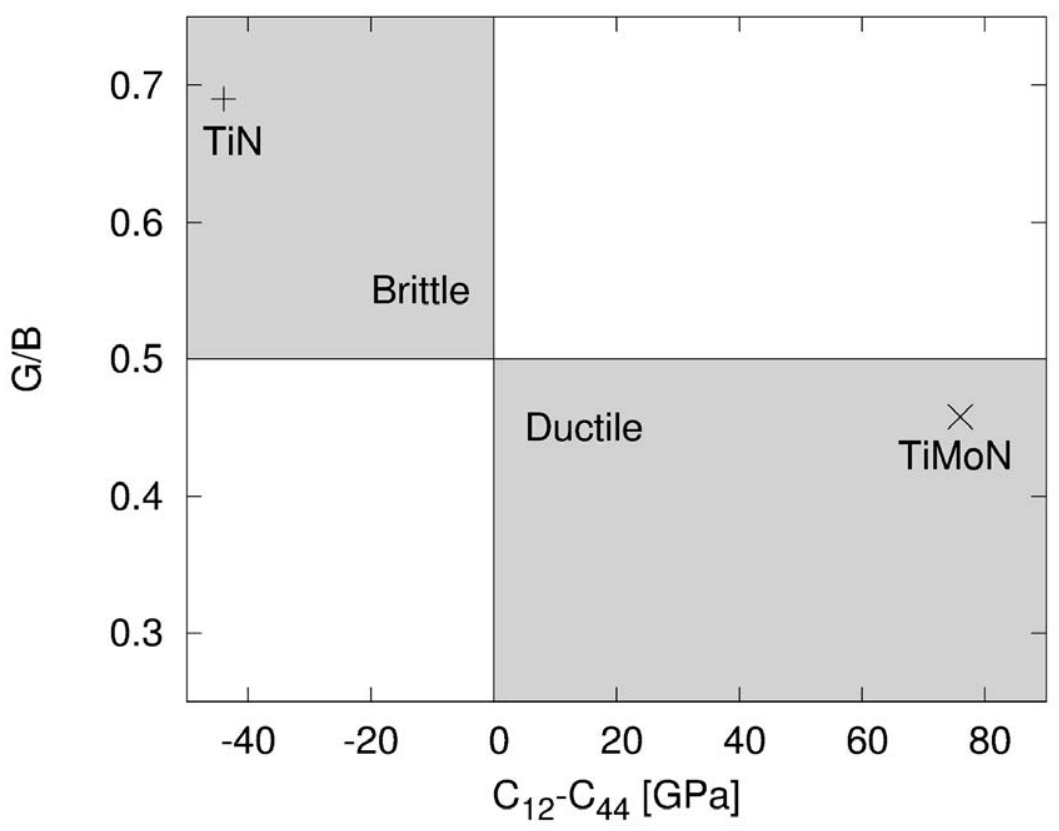

Figure 3.1: Example of a brittle/ductile map based on the Pugh and Pettifor criteria for ductility. The region associated with brittleness is highlighted in light red and the region associated with ductility in light blue. A brittle material, TiN, and a ductile material, TiMoN, have been included. 


\subsection{Calculation of stress/strain curves}

While the elastic constants provide useful information about the mechanical behavior of a material, they are limited to deformations small enough for Hooke's law to be valid. To obtain information about the material response beyond the elastic regime, calculations of stress/strain curves are required.

Stress/strain curves show the stress within a material as it is progressively strained. As long as the strain is within the elastic regime, the stress will follow a linear behavior, as discussed previously, while for larger strains deviations from the linear behavior appear and slips may occur.

The stresses are calculated by displacing a family of lattice planes along a specified direction. Which planes are displaced and along which direction the displacement occurs specifies a slip system. For cubic transition metal nitrides and carbides, the $\{110\}\langle 1 \overline{1} 0\rangle$ and $\{111\}\langle 1 \overline{1} 0\rangle$ slip systems are known to be active $[47,48]$. Therefore, the calculations of stress/strain curves carried out in paper 7 are limited to these systems. The stress/strain curves calculated within are obtained by shearing the supercell in strain intervals of $1 \%$, and taking the stress directly from the output of the calculations.

\subsection{Atomic ordering}

The transition metal nitrides and carbides studied in this work crystallize in the rock-salt structure, which can be viewed as two interlocking face-centered cubic crystal lattices. These two lattices are referred to as the metal and nitrogen, or carbon for the carbides, sublattices. When alloying two, or more, materials of this family the different metals can be arranged in many different ways on the metal sublattice. How the degree of ordering influences the properties of these materials is one question studied within this thesis. To this end, five different arrangements have been investigated, three of which are ordered and two of which are disordered.

The ordered structures are the $\mathrm{C \# 1}, \mathrm{C} \# 3$, and $\mathrm{CuPt}$ structures, shown in Figure 3.2. The $\mathrm{CH1}$ structure consists of alternating $M^{l}$ and $M^{2}$ planes in the [001] direction, whereas the alternating planes of the CuPt structure are along [111] direction. The $\mathrm{C \# 3}$ structure is similar to the $\mathrm{CuPt}$ structure, in that (111) planes alternatingly contain $75 \% M^{I}$ and $25 \%$ $M^{2}$, and $25 \% M^{l}$ and $75 \% M^{2}$. Regarding these three ordered structures, it is worth noting that only the $\mathrm{C \# 3}$ structure obeys the cubic symmetry. The $\mathrm{C \# 1}$ structure is tetragonal, and the CuPt structure is rhombohedral. Additionally, the $\mathrm{C \# 1}$ and $\mathrm{C \# 3}$ structures correspond to the maximum and minimum number of $M^{1}-M^{2}$ bonds, respectively [49]. 
(a)

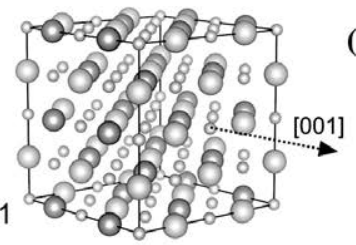

(b)

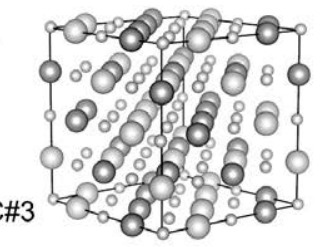

(c)

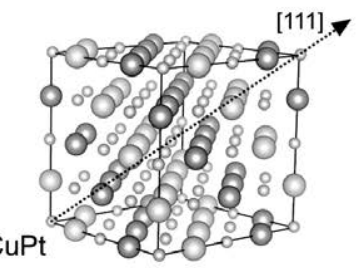

Figure 3.2: Ordered structures on the metal sublattice. (a) $\mathrm{C \# 1}$ structure, (b), $\mathrm{C \# 3}$ structure, and (c) $\mathrm{CuPt}$ structure.

The first of the disordered structures is one in which the metal atoms are randomly distributed on the metal sublattice. Initially, it may seem that this corresponds to a fully disordered structure. Due to the periodic boundary conditions of the calculation, this is however not the case. To model a disordered solid it is instead advisable to use the Special Quasi-random Structure-method (SQS) [50], which is the second disordered structure used herein. The two disorded structures are shown in Figure 3.3.

(a)

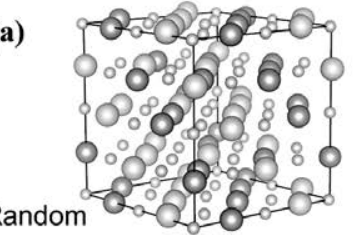

(b)

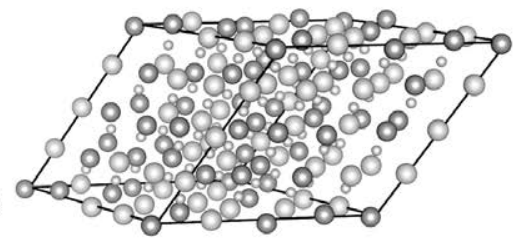

Figure 3.3: Disordered structures. (a) Random assignment of metal atoms, with intermediate disorder. (b) Fully disordered SQS.

A disordered structure is one in which the correlation between atomic sites is zero. To put it in another way, the probability of finding an atom of type $M 2$ at a given distance away from an atom of type $M 1$ should be exactly equal to the concentration of $M 2$ in the material. This is quantified in the Warren-Cowley short-range order parameter [51], defined as

$$
\alpha_{n}=1-\frac{P_{M^{2}}\left(R_{n}\right)}{x}
$$

Here, $\alpha_{n}$ is the Warren-Cowley parameter for shell $n, P_{M^{2}}\left(R_{n}\right)$ the probability of fidning an atom of type M2 at distance $R_{n}$ from an atom of type M1, and x the concentration of M2. For an ideal solid solution, this parameter will then equal zero on all shells. The SQS method is based on designing structures in such a way as to make as many as these parameters equal zero. The Warren-Cowley parameters for the structures presented here are shown in Figure 3.4. 


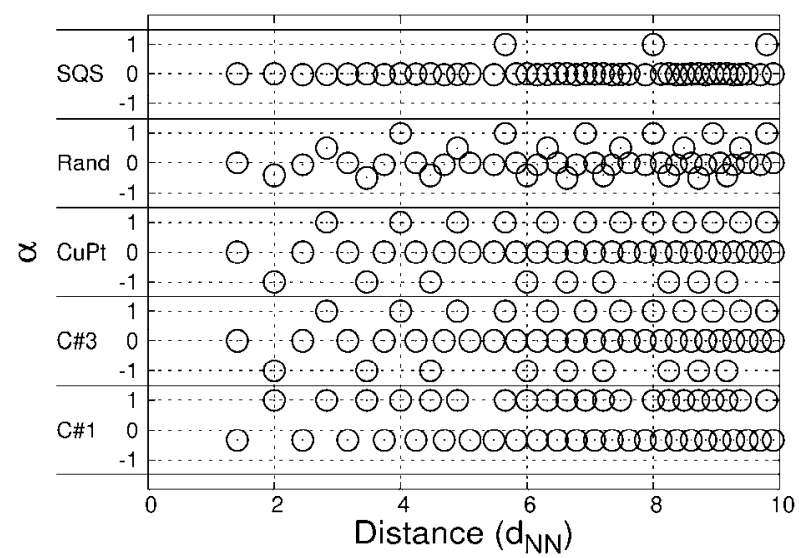

Figure 3.4: Warren-Cowley short-range order parameters for ordered and disordered structures.

\subsection{Thermodynamical stability}

The second law of thermodynamics states that the entropy of an isolated system is at a maximum at equilibrium. For a system at constant temperature and pressure, this translates to a minimum in Gibbs' free energy

$$
G=E-T S+p V
$$

where $E$ is the internal energy, $T$ and $S$ are conventional temperature and entropy, respectively, $p$ is pressure, and $V$ is volume. A common approach is to perform DFT calculations at a temperature of $0 \mathrm{~K}$ and ideal volume, in which case only the internal energy contributes to $G$. The energy of mixing of a $M_{x}^{1} M_{1-x}^{2}$ alloy is then defined as the difference in internal energy of the alloy compared to the weighted average of internal energies of the alloying materials. Expressed mathematically,

$$
\Delta E_{\text {mix }}=E_{M_{1-x}^{1} M_{x}^{2}}-(1-x) E_{M^{1}}-x E_{M^{2}},
$$

where $x$ is the fraction of material $M^{2}$ and (1-x) the fraction of material $M^{1}$. If $\Delta E_{\text {mix }}$ is negative, the alloy is thermodynamically stable with respect to phase separation into its constituent materials, whereas a positive $\Delta E_{\text {mix }}$ indicates instability. A $\Delta E_{\text {mix }}$ close to zero may indicate metastability, where the thermodynamic driving force for phase separation is negated by kinetic barriers. Since PVD techniques are off-equilibrium processes, they are especially suited for deposition of metastable phases.

At non-zero temperatures, entropic effects also contribute to Gibbs' free energy. These contributions are challenging for first-principle techniques, as they require computationally demanding techniques such as ab-initio molecular dynamics or Monte 
Carlo methods. A first approximation is to include the effect of configurational entropy, which can be approximated using a mean-field approach as

$$
-T S_{\text {conf }}=k_{B} T(x \ln (x)+(1-x) \ln (1-x)),
$$

Since $0<x<1$, this contribution will lower Gibbs' free energy as the temperature is increased, and thereby stabilize the solid solution. 


\section{MD Simulations of Film Growth}

\subsection{Thin film deposition}

Thin films are materials with thicknesses ranging from a few atomic layers, on the order of nanometers, to micrometers. Several techniques exist by which thin films may be deposited on a substrate, grouped into the categories of chemical and physical vapor deposition. The work in this thesis is focused on the simulation of processes related primarily to magnetron sputtering, which is briefly described here. A sputtering deposition system consists of a vacuum chamber in which there is a substrate on which the thin film is to be deposited, a target acting as source of the material that is to be sputtered, and a sputtering gas, for example argon.

By applying a negative voltage to the target, the sputtering gas is ionized and the positive ions are attracted to the target. When the ions bombard the target, their kinetic energy is transferred to the atoms in the target, which are knocked out and travel toward the substrate where they condense to form the thin film. A schematic of a sputtering deposition process is shown in Figure 4.1.

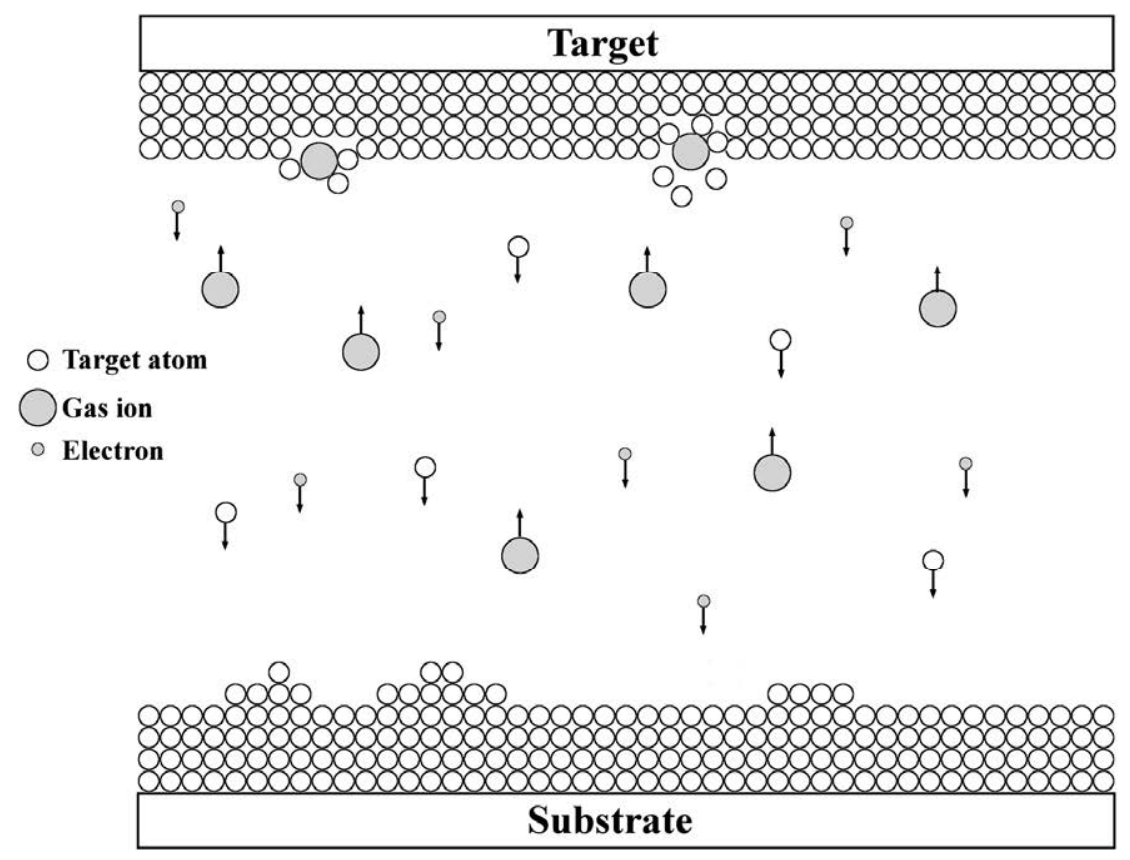

Figure 4.1: Schematic illustration of a sputtering deposition process. An applied electric field ionizes the sputtering gas, creating positively charged ions (light red) and free electrons (light blue). The gas ions are accelerated towards the target, where they knock out target atoms. The target atoms travel toward the substrate where they condense and form the growing film. 
Sometimes the sputtering system contains a reactive gas that also participates in forming the thin film. For example, thin films of TiN are typically deposited using targets of $\mathrm{Ti}$ and a gas mixture containing $\mathrm{N}_{2}$. The fraction of $\mathrm{N}_{2}$ in the gas mixture, often expressed in terms of partial pressure, is an important parameter in the film growth process.

It is also possible to apply an electric bias to the substrate and thereby accelerate electrically charged species toward the substrate surface, which is another parameter that may significantly affect the structure of the grown film.

\subsection{Film microstructure and growth modes}

During thin film growth, atoms and molecules condense on the substrate to form the growing film. These adatoms and admolecules, or adspecies to call them in one word, diffuse on the substrate surface, interacting with the substrate and with each other. As adspecies come into contact with each other, they form larger clusters which may also be mobile. At some critical size, the clusters cease to be mobile and remain stationary on the substrate surface. These clusters serve as nucleation sites for the growing film, and will continue to grow as adspecies nucleate upon them. As the growth process continues and the nucleating clusters grow, they come into contact with each other and coalesce, thereby forming larger clusters and eventually forming a continuous film. The way by which the film grows is broadly categorized in three categories, illustrated in Figure 4.2.

(a)

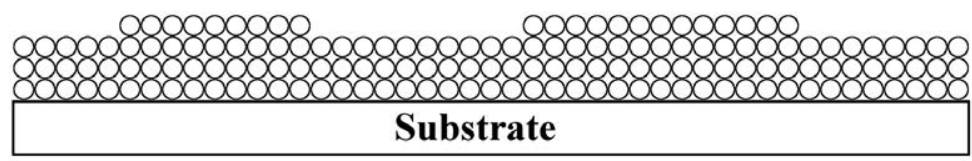

(b)

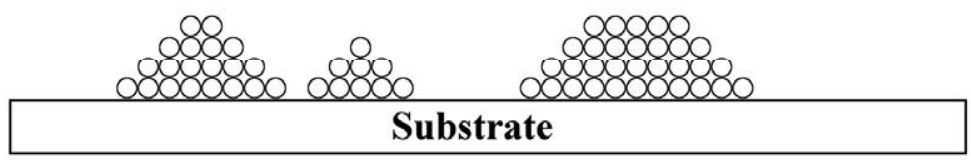

(c)

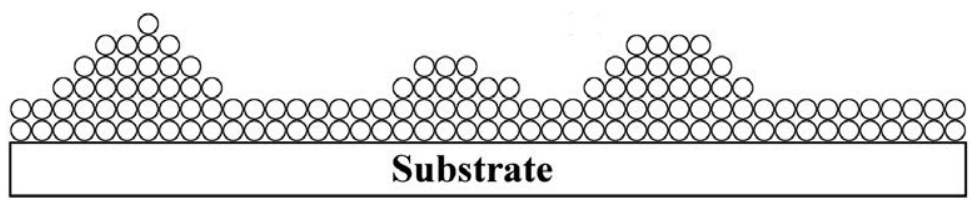

Figure 4.2: Schematic illustration of thin film growth modes. (a) Frank-van der Merwe growth, (b) Volmer-Weber growth, and (c) Stranski-Krastanov growth. 
If the interaction between the adspecies and the substrate is stronger than the interaction between different adspecies, the film tends to grow one flat atomic layer at a time. This is known as Frank-van der Merwe growth, or layer-by-layer growth. The opposite is the case where the interaction between different adspecies is stronger than the interaction between the substrate and adspecies. This growth mode, known as VolmerWeber growth, is characterized by the formation of 3D islands growing separately. Stranski-Krastanov growth occurs if the interactions between substrate and adspecies is of a similar strength to the strength between different adspecies, and is characterized by layerby-layer growth for the initial layers which evolves into island growth for subsequent layers.

The effect of the substrate during thin film growth is to impose its symmetry on the growing film, as the arriving atoms are driven to sites minimizing the potential energy. The process of a growing film obeying the crystal symmetry of the substrate is known as epitaxy. However, many other parameters affect the structure of the film, and different structures may be obtained by varying deposition parameters such as substrate temperature and incident energy. The relation between deposition parameters and film microstructure can be qualitatively visualized in a structure zone diagram [52].

\subsection{Molecular Dynamics studies of thin film growth}

Typically, the parameters of a deposition process are tuned using trial and error to achieve the desired film structure and properties. The reason for this is an overall lack of insight into the dynamics of atomic-scale mass transport and their effects on nucleation and growth. This lack of insight, in turn, stems from the inability of even the most advanced experimental techniques, such as low energy electron microscopy [16,17] and scanning tunneling microscopy [13-15] to resolve the pico-second timescale at which these processes occur.

By employing computational techniques, primarily MD simulations, details regarding diffusion pathways, nucleation, coalescence, and descent mechanisms from islands, all of which significantly affect thin film growth, can be extracted. The use empirical potentials, however, limits the applicability of CMD to well-studied systems for which interaction potentials can be developed.

Within the family of transition metal nitride coatings, TiN is one of the most explored. Not only bulk physical properties, but also nucleation, growth, and surface morphological evolution of TiN surfaces have been studied with a variety of techniques. With this data available, TiN interaction potentials have been developed and systematically 
optimized for the study of nucleation and diffusion. Due to the general structural and chemical similarities between TiN and other transition metal nitrides, it is expected that the results of these studies will apply to most transition metal nitride surfaces.

\subsection{Diffusion on infinite TiN(001) terraces}

In building an understanding of the atomic-scale processes dictating thin film growth, a reasonable starting point is diffusion on infinite terraces. Experimental and theoretical studies have shown that the primary diffusing species on $\operatorname{TiN}(001)$ during growth are Ti and $\mathrm{N}$ adatoms, as well as $\mathrm{TiN}_{\mathrm{x}}$ admolecules, where $\mathrm{x}$ ranges from 1 to 3 $[19,20]$.

I find that Ti adatoms favor adsorption at fourfold hollow sites, surrounded by two Ti and two $\mathrm{N}$ terrace atoms. My initial studies showed that $\mathrm{N}$ adatoms also favor the fourfold hollow site, but a more detailed investigation revealed that these sites are metastable, and the stable site is actually the threefold site [53]. The adsorption energy of a $\mathrm{N}$ adatom on the $\operatorname{TiN}(001)$ surface in this position is higher (less negative) than the binding energy of an $\mathrm{N}$ atom in a $\mathrm{N}_{2}$ molecule, indicating that $\mathrm{N}$ is much more stable in the gas phase than adsorbed on the TiN(001) surface. Should two N adatoms come into contact, they should thus bond to form $\mathrm{N}_{2}$ and desorb. This was also observed in the simulations here.

Ti adatoms diffuse mainly via [100] and [010] channels, and very rarely via $<110>$ channels, despite a calculated energy difference of only $0.1 \mathrm{eV}$ between the two barriers. The discrepancy can be understood as a temperature effect, as $\mathrm{N}$ terrace atoms have a large vibrational amplitude and hinder Ti diffusion along $<110>$. N adatoms, however, diffuse along $<110>$ channels via metastable positions atop Ti terrace atoms. Ti adatoms also diffuse significantly more than $\mathrm{N}$ adatoms, with double the diffusion coefficient.

According to static DFT calculations, the stable configurations of $\mathrm{TiN}_{\mathrm{x}}$ molecules are with all molecule atoms in epitaxial sites. CMD simulations at $1000 \mathrm{~K}$ however, show that the stable configurations for $\mathrm{TiN}$ and $\mathrm{TiN}_{2}$ are with the $\mathrm{Ti}$ atom in a fourfold hollow site and the $\mathrm{N}$ atoms in neighboring epitaxial sites. The $\mathrm{TiN}_{3}$ molecule is found to favor a $\mathrm{T}$-shaped configuration with all atoms in epitaxial sites. However, one $\mathrm{N}$ atom frequently alternates between an epitaxial site and a fourfold hollow site, thus reshaping the molecule into a Y-shape.

The diffusion mechanisms of $\mathrm{TiN}_{\mathrm{x}}$ molecules can be significantly more complex than those of adatoms, as the internal structure of the molecules allows for both rotation and translation, which are observed for all admolecules. TiN molecules display an 
additional mechanism, dubbed roto-translation, which is a combination of two rotations and a translation. This mechanism is showed in Figure 4.3.

(a)

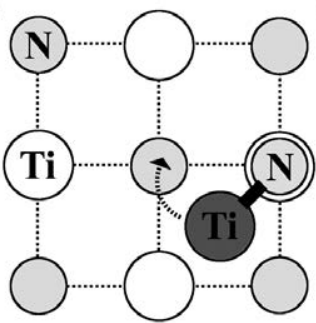

(c)

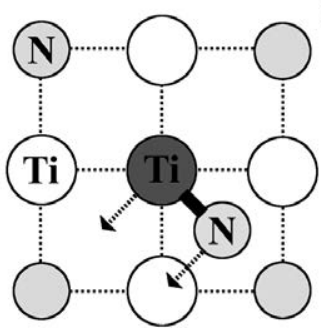

(b)

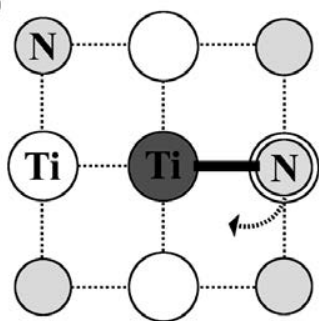

(d)

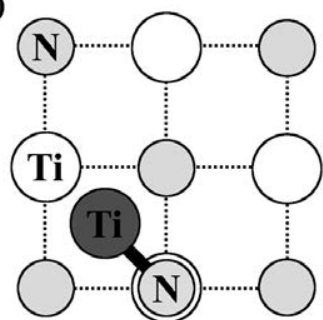

Figure 4.3: Roto-translation of TiN dimer. (a) From the stable configuration, the Ti dimer atom moves on top of a terrace $\mathrm{N}$ atom. (b) The $\mathrm{N}$ dimer atom moves into the vacated fourfold hollow site. (c) The Ti and $\mathrm{N}$ dimer atoms translate concertedly into (d) a new stable configuration.

As $\mathrm{x}$ is increased from 1 to 3 the frequency of rotation events increases while the frequency of translations decreases. DFT calculations show that the interaction between the $\mathrm{Ti}$ atom in a $\mathrm{TiN}_{2}$ molecule and a terrace $\mathrm{Ti}$ atom is much weaker than the corresponding interaction for a $\mathrm{TiN}$ molecule, causing rotation to become easier to initiate. Rotation of a $\mathrm{TiN}_{3}$ molecule, however, leads to no mass transport. Consequently, $\mathrm{TiN}_{3}$ molecules are essentially stationary on $\mathrm{TiN}(001)$ and serve as the smallest nucleating clusters.

\subsection{Diffusion on and descent from islands}

Diffusion rates and mechanisms on islands may differ significantly from those on infinite terraces, as relaxation of the island results in shorter interatomic distances between island atoms. This is especially noticeable for small islands, since the structure of large islands approaches that of the terrace. Additionally, diffusion over an island edge, or descent from islands, may be hindered by a significant energy barrier. This barrier, known as the Ehrlich-Schwoebel barrier, arises from the descending species having to break bonds upon descending from the edge of the island. 
On square $8 \times 8$ atoms, two-dimensional TiN/TiN(001) islands, the diffusion paths of $\mathrm{Ti}$ and $\mathrm{N}$ adatoms do not differ from those on an infinite terrace. However, the difference in diffusion rates is notably increased as Ti adatoms experience a significant funneling and rapidly reach the edge, whereas $\mathrm{N}$ adatoms require longer times to reach the edge.

Having reached the edge, $\mathrm{Ti}$ adatoms descend from the island edge by means of push-out/exchange, a mechanism which is illustrated in Figure 4.4.
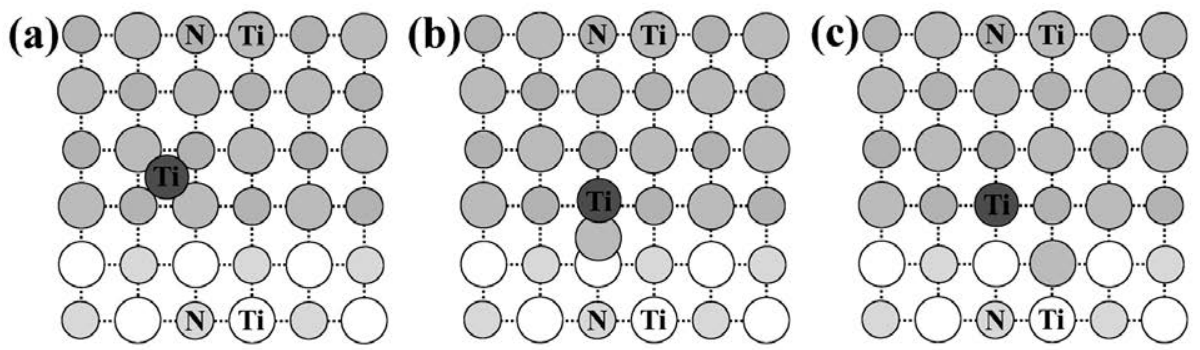

Figure 4.4: Ti push-out/exchange at island edge. (a) Ti adatom in fourfold hollow at island edge. (b) Ti at bridge position at the island edge, beginning to push out the underlying Ti atom. (c) Completion of push-out/exchange event.

By pushing out a Ti edge atom and occupying the newly vacated site, the EhrlichSchwoebel barrier is bypassed. Calculations using the nudged elastic band (NEB) method confirms that the energy barrier for push-out/exchange is lower than for direct hopping over the edge.

$\mathrm{N}$ adatoms are observed to descend both via direct hops over the island edge and via push-out/exchange, depending on the region of the island where the descent occurs. NEB-calculations indicate that direct hopping is energetically favored at all positions, contradicting the observations. However, at finite temperatures, direct hopping is further hindered by the oscillations of island atoms.

The diffusion of TiNx admolecules on TiN/TiN(001) islands proceeds mainly along the same channels as on infinite terraces. However, TiN displays an additional diffusion mechanism not seen on terraces, here dubbed articulated roto-translation, in which the translation is parallel to the dimer bond axis.

Both TiN and $\mathrm{TiN}_{2}$ molecules are highly mobile on islands, with average residence times even lower than that of Ti adatoms. TiN dimers display several descent mechanisms, including direct hopping, push-out exchange, and a combination of the two where the Ti dimer atom pushes out an underlying $\mathrm{Ti}$ atom and the $\mathrm{N}$ dimer atom hops over the island edge. $\mathrm{TiN}_{2}$ trimers only descend via direct hopping over the island edges. Interestingly, 
$\mathrm{TiN}_{2}$ molecules can be trapped if they reach $\mathrm{N}$ islands corners, where the strong interaction between the $\mathrm{N}$ corner atom and the Ti trimer atom prevents further diffusion.

$\mathrm{TiN}_{3}$ molecules are essentially stationary on $\mathrm{TiN} / \mathrm{TiN}(001)$ islands, as they are on infinite terraces. In general, then, the formation of $\mathrm{TiN}_{3}$ molecules on terraces as well as on islands lead directly to island growth and promote multilayer growth. Ti adatoms and $\mathrm{TiN}_{2}$ molecules, however, rapidly descend and thus promote two-dimensional growth.

\subsection{Effect of N/Ti flux ratios on TiN growth}

The results of the studies of the dynamics of $\mathrm{Ti}$ and $\mathrm{N}$ adatoms and $\mathrm{TiN}_{\mathrm{x}}$ admolecules detailed above indicate that the N/Ti flux ratio should have a significant effect on the growth modes of TiN films by altering the relation between the amounts of different adspecies. To investigate the effect of the N/Ti ratio during deposition, large scale CMD simulations of $\mathrm{TiN}$ deposition on $\mathrm{TiN}(001)$ surfaces were carried out using incident N/Ti flux ratios of 1, 2 and 4 . In a reactive sputtering deposition process, increasing the N/Ti flux ratio corresponds to increasing the $\mathrm{N}_{2}$ partial pressure.

With $\mathrm{N} / \mathrm{Ti}=1$, the resulting film is globally understoichiometric and features small metal-rich 111-oriented regions. The 111-oriented grains form due to local $\mathrm{N}$ deficiency, as excess metal atoms occupy non-epitaxial sites and serve as seeds for 111 oriented growth. Increasing the N/Ti flux ratio suppresses the formation of these regions, which is completely eliminated with $\mathrm{N} / \mathrm{Ti}=4$.

Variations in the incident $\mathrm{N} / \mathrm{Ti}$ flux ratio also translate into changes in the islands edge orientations. With $\mathrm{N} / \mathrm{Ti}=1$, both 100 and polar 110-oriented island edges are formed, with the former being in the majority. Increasing N/Ti results in an increase of $\mathrm{N}$ terminated polar 110 island edges, as the excess nitrogen is accommodated at the island edges. As a result, the films grown with $\mathrm{N} / \mathrm{Ti}=2$ and 4 are globally overstoichiometric, but locally stoichiometric.

The simulations show that an insufficient $\mathrm{N} / \mathrm{Ti}$ flux ratio leads to surface roughening via the nucleation of 111 oriented islands, as described above, whereas high $\mathrm{N} / \mathrm{Ti}$ ratios result in surface roughening due to more rapid upper-layer nucleation. At high $\mathrm{N} / \mathrm{Ti}$ ratios, more stationary $\mathrm{TiN}_{3}$ admolecules are formed, which act as nucleating clusters. $\mathrm{N} / \mathrm{Ti}=2$ results in close to layer-by-layer growth.

Vacancy formation is also significantly affected by the N/Ti flux ratio. Increasing the N/Ti ratio from 1 to 4 shifts the vacancy formation from primarily N-vacancy formation to Ti-vacancy formation. Deposition with $\mathrm{N} / \mathrm{Ti}=2$ results in overall fewer vacancies than with either $\mathrm{N} / \mathrm{Ti}=1$ or $\mathrm{N} / \mathrm{Ti}=4$. Increasing the $\mathrm{N} / \mathrm{Ti}$ ratio increases the likelihood of $\mathrm{Ti}$ 
atoms migrating from the substrate surface into the deposited layer, whereas $\mathrm{N}$ lost due to $\mathrm{N}_{2}$ desorption is more easily replaced by excess $\mathrm{N}$ in the deposition flux.

\subsection{Effect of incident $\mathrm{N}$ energy on TiN growth}

TiN film growth can also be tailored by applying a substrate bias, and thereby accelerating charged particles onto the substrate. The effect of this is investigated using CMD simulations at different incident $\mathrm{N}$ kinetic energies $\mathrm{E}_{\mathrm{N}}$. Two different energies are used: $E_{N}=2 \mathrm{eV}$ and $\mathrm{E}_{\mathrm{N}}=10 \mathrm{eV}$. A third case is constructed by initially using $\mathrm{E}_{\mathrm{N}}=2 \mathrm{eV}$ and setting it to $10 \mathrm{eV}$ after two thirds of the total simulation time. In all three cases a flux ratio of $\mathrm{N} / \mathrm{Ti}=4$ is used.

With $\mathrm{E}_{\mathrm{N}}=2 \mathrm{eV}$, the resulting film is globally overstoichiometric with islands bounded by $\mathrm{N}$-termined 110 edges, and nucleating in the upper layers of the film. Islands tend to grow in branching structures with a high edge-length to area ratio.

In contrast, with $\mathrm{E}_{\mathrm{N}}=10 \mathrm{eV}$, a smaller fraction of the incident $\mathrm{N}$ is retained, and as a result the grown films are very close to ideal stoichiometry; in this case islands grown have both 100 and 110 oriented edges. Additionally, islands grow more compact than for $E_{N}=2 \mathrm{eV}$, as exhibited by a lower edge-length to area ratio. However, at this energy, the formation of a 111 oriented region, as for low N/Ti flux ratios at $\mathrm{E}_{\mathrm{N}}=2 \mathrm{eV}$, is observed.

The type of vacancies formed is also significantly altered by the incident $\mathrm{N}$ kinetic energy. With $\mathrm{E}_{\mathrm{N}}=2 \mathrm{eV}$, only Ti vacancies exist in the film, as any $\mathrm{N}$ loss due to desorption is easily replaced by the excess $\mathrm{N}$ in the deposition flux. For $\mathrm{E}_{\mathrm{N}}=10 \mathrm{eV}$ however, the $\mathrm{N}$ incorporation probability is significantly reduced, and as a result the majority of vacancies reside on the $\mathrm{N}$ sublattice.

Switching the incident $\mathrm{N}$ energy from 2 to $10 \mathrm{eV}$ during growth results in films which overall are more compact than those grown with $\mathrm{E}_{\mathrm{N}}=2 \mathrm{eV}$, and which have significantly lower concentrations of adatoms, admolecules and small islands. The branching islands structure seen for $\mathrm{E}_{\mathrm{N}}=2 \mathrm{eV}$ is eliminated after increasing the energy to $10 \mathrm{eV}$. Furthermore, there is no formation of 111-oriented regions as is the case for deposition with $\mathrm{E}_{\mathrm{N}}=10 \mathrm{eV}$. The amount of vacancies in the film is also significantly reduced throughout compared to the depositions with $2 \mathrm{eV}$ or $10 \mathrm{eV}$.

\subsection{Visualization of diffusion and film growth}

Several of the phenomena mentioned above feature complex sequences of motion, including simultaneous rotation and vibration of several atoms, which are difficult to describe using static 2D images. Scientific visualization is emerging as an interdisciplinary 
field, dedicated to using modern computer graphics to display scientific data in a manner which enables scientists to interpret and understand otherwise overwhelming data.

I have recorded several movies of diffusion and descent phenomena, as well as segments of film growth simulations. These are available to download from https://www.dropbox.com/sh/uw366xgdtk6fkba/AABbh-y0ylnBR0vvbJzkErxwa?dl=0, and free to share provided acknowledgment is given to: D. Edström and D. G. Sangiovanni; Department of Physics (IFM); Linköping University. Web site and contents are subject to change.

The movies available show, in detail, the diffusion mechanisms of $\mathrm{Ti}$ and $\mathrm{N}$ adatoms as well as $\mathrm{TiN}, \mathrm{TiN}_{2}$, and, $\mathrm{TiN}_{3}$ admolecules. The movies are particularly helpful for the understanding of the more complex pathways of $\mathrm{TiN}_{\mathrm{x}}$ admolecules. Also available are time-lapse movies of TiN film growth at different $\mathrm{N}$ energies $\mathrm{E}_{\mathrm{N}}$ and $\mathrm{N} / \mathrm{Ti}$ flux ratios, which allow the viewer to track the evolution of the film structure over time in a manner difficult to convey with static images. Examples of high-resolution movies of the initial stages of growth are also provided, which allow for the interested viewer to see the formation of the initial seeds for $2 \mathrm{D}$ and $3 \mathrm{D}$ growth. 


\section{Mechanical properties of transition metal nitrides and carbides}

\subsection{Ceramic protective coatings}

Ceramic materials such as transition metal nitrides and carbides are frequently used as protective coatings to improve performances and lifetimes of components in a wide variety of applications, for example cutting tools and engine components.

To increase the performance of ceramic protective coatings, a significant amount of research has been invested into improving their hardness. However, increased hardness is generally accompanied by increased brittleness, leading to cracking during extreme working conditions and the coatings subsequently being peeled off from the component they were employed to protect. It is therefore necessary to design coatings which, aside from maintaining hardness, also exhibit increased ductility. In a ductile material, crack propagation is hindered by plastic flow.

\subsection{Toughness enhancement in transition metal nitride alloys}

One approach to tailor the properties of coatings is to create alloys of two or more materials. For ternary TMN alloys, it has been shown that tuning the valence electron concentration via the selection of alloying elements, allows for tailoring of the Cauchy pressure and $\mathrm{G} / \mathrm{B}$ ratio $[11,12]$, and the design of ductile materials.

From an electronic structure point of view, the increase in ductility upon alloying is explained as an effect of optimizing the occupancy of shear-sensitive $d$ - $t_{2 g}$ bonding states. In this way, the formed alloy is more compliant to shear while retaining the strength of the metal-N bonds as found in the parent binary compounds.

Upon shearing of the VEC-optimized ternary alloys, a layered electronic structure is formed, where alternating planes of high and low charge are formed perpendicularly to the strain direction. Dislocation glide is initiated at the interface between these planes, allowing for the dissipation of mechanical stress.

The theoretical predictions of increased toughness in ternary TMN alloys were verified experimentally for $\mathrm{V}_{0.5} \mathrm{Mo}_{0.5} \mathrm{~N}(001)$ thin films deposited on $\mathrm{MgO}(001)$ substrates [54]. Nanoindentation tests show that while TiN and $\mathrm{VN}$ thin films crack, $\mathrm{V}_{0.5} \mathrm{Mo}_{0.5} \mathrm{~N}$ do not. Instead, $\mathrm{V}_{0.5} \mathrm{Mo}_{0.5} \mathrm{~N}$ exhibit material pile-up, characteristic of plastic flow.

\subsection{The effect of atomic ordering on mechanical properties of TMN alloys}

The theoretical calculations carried out for the VEC-optimized ternary alloys described above were all limited to highly ordered structures. In order to determine the 
influence of atomic ordering, and whether the improvements in ductility observed for ordered structures also hold for disordered structures, calculations of the elastic properties of $M^{l} M^{2} \mathrm{~N}$, where $M^{1}=\mathrm{Ti}$ or $\mathrm{V}$, and $M^{2}=\mathrm{W}$ or Mo, with different degrees of order were carried out.

The resistance to shear deformation is considerably reduced upon alloying for all structures, ordered as well as disordered. Ordered structures do exhibit lower values of $\mathrm{C}_{44}$ elastic constant and higher values of bulk modulus and the $\mathrm{C}_{11}$ elastic constants than disordered structures, indicative of lower resistance to shear deformation and higher resistance to tensile deformation, respectively.

Upon shearing, ordered structures form a layered electronic structure with fully bonding metallic states perpendicular to the applied stress, as discussed above. The effect is not observed in disordered structures. Instead, metallic bonds are formed between every second neighboring $\mathrm{Me}-\mathrm{Me}$ pair, resulting in a partially bonding metallic state. This effect is illustrated in Figure 5.1.
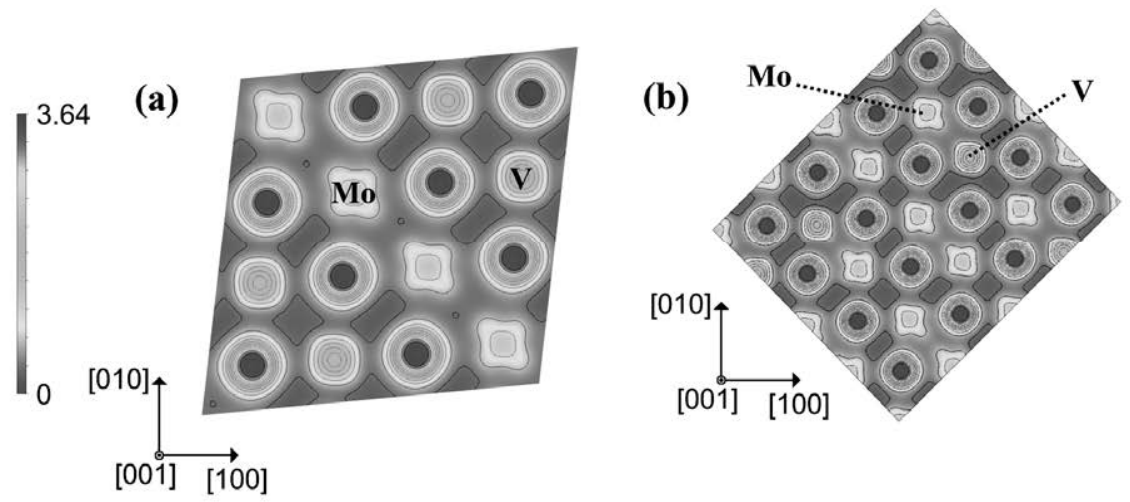

Figure 5.1: Charge densities of sheared (a) C\#3 (ordered) and (b) SQS (disordered) VMoN. Shearing of ordered VMoN results in a layered structured with fully bonding metallic states perpendicular to the applied stress. Shearing of disordered VMoN results in the formation of metallic pairs in the direction perpendicular to the applied stress. Charge densities are expressed in $\mathrm{e}^{-} / \AA^{3}$.

Evaluating the ductility of the alloys using the Pugh and Pettifor criteria, displayed in Figure 5.2, shows that variations in ordering induces variations in the Cauchy pressure and $\mathrm{G} / \mathrm{B}$ ratios within a limited region, demonstrating that the increased toughness in these alloys are primarily an effect of the increased valence electron concentration. 


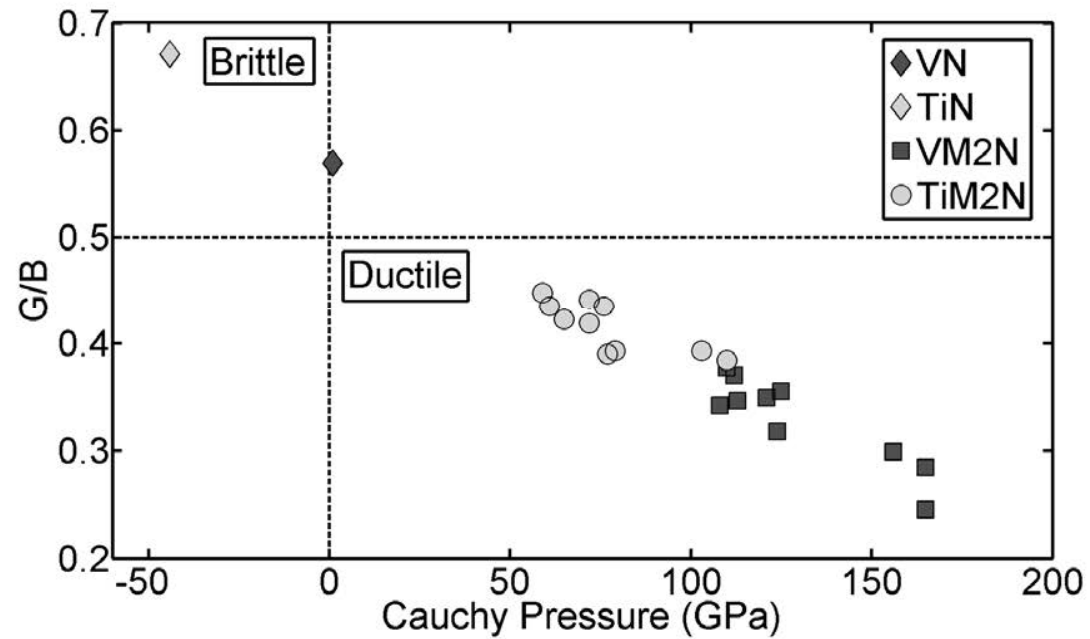

Figure 5.2: Brittle/ductile map of $M^{1} M^{2} \mathrm{~N}$ alloys of different ordering on the metal sublattice. Cauchy pressure and $G / B$ vary slightly as the ordering on the metal sublattice is changed, but are primarily determined by the valence electron configuration.

\subsection{Elastic properties of ternary transition metal carbide alloys}

Transition metal carbides and nitrides share many similarities in structure and properties, owing to the similarities between the electron configurations of $\mathrm{C}$ and $\mathrm{N}$ atoms. It can therefore be expected that VEC tuning can be used to enhance the ductility of TMC alloys as for TMN alloys. Nevertheless, theoretical investigations, such as those that were carried out for TMN alloys, are needed to verify this hypothesis.

It should be noted that VEC tuning has previously been employed to increase hardness in TMC alloys. For example, Hugosson et al [9] used VEC tuning to set the energies of the cubic and hexagonal phases equal, thereby promoting the formation of stacking faults which hinder dislocation glide, resulting in increased hardness. It has also been theoretically shown that hardness reaches a maximum for $\mathrm{VEC}=8.4$ in cubic transition metal carbo-nitrides [8], as shear-resistant metal-N bonds are completely occupied. The question that is attempted to be answered here is whether VEC tuning can also be used to promote ductility in TMC alloys, as was shown to be the case for TMN alloys.

Calculations of the elastic properties of select $M^{l} M^{2} \mathrm{C}$ alloys show that alloying of these materials in general leads to an increase in bulk modulus, indicative of enhanced incompressibility and hardness. However, the analysis according to the Pugh and Pettifor criteria, presented in Figure 5.3, predicts no enhanced ductility in these alloys. 


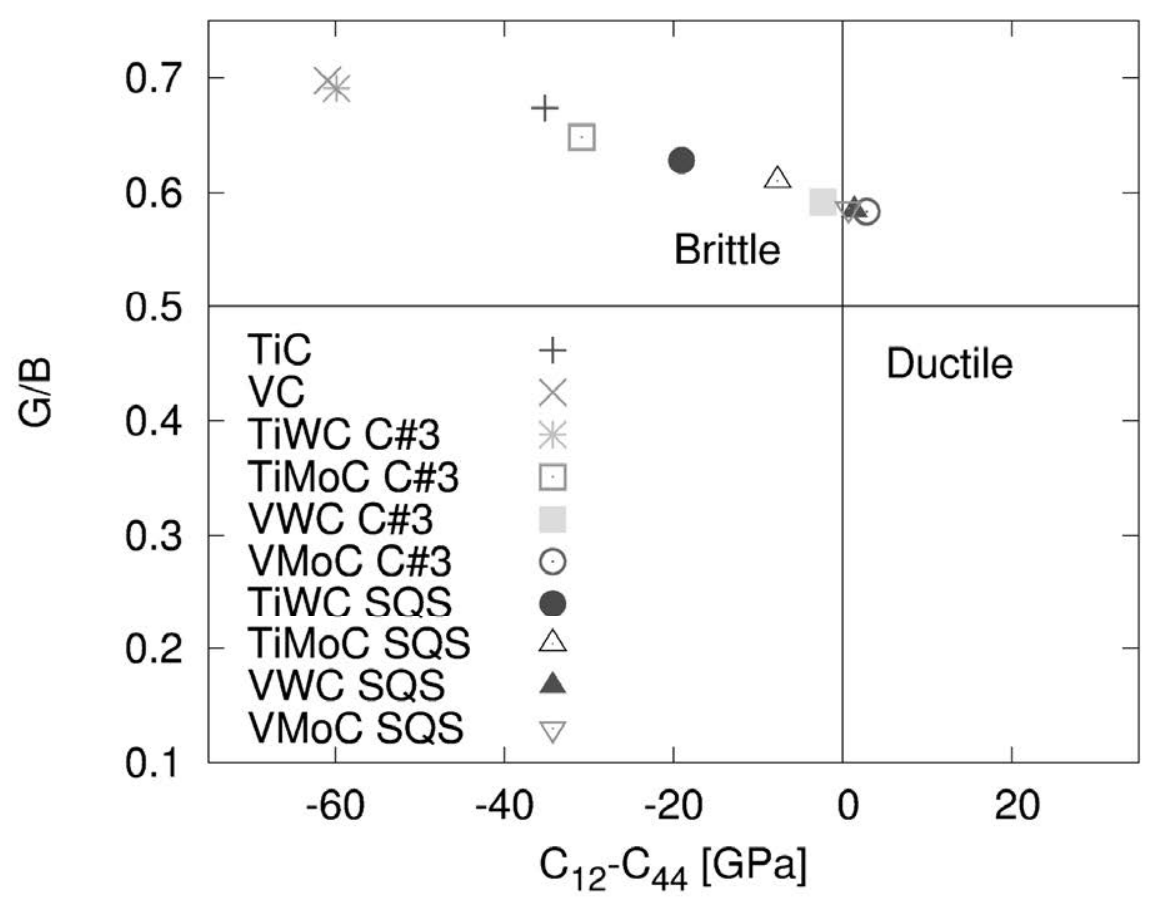

Figure 5.3: Brittle/ductile map of studied ternary carbide alloys. According to the criteria of Pugh and Pettifor, none of the investigated alloys are predicted to exhibit ductile behavior.

The calculated Cauchy pressures of the $\mathrm{V} M^{2} \mathrm{C}$ alloys are comparatively close to zero, between -2 and $3 \mathrm{GPa}$, and are thus close to the limit of the Pettifor criterion. A further analysis is carried out by calculating the stress/strain curves along the $\{110\}\langle 1 \overline{1} 0\rangle$ and $\{111\}\langle 1 \overline{1} 0\rangle$ slip systems. The results for the $\{111\}\langle 1 \overline{1} 0\rangle$ slip system are shown in Figure 5.4. While the majority of the materials exhibit linear stress/strain curves, characteristic of an elastic response, the stress in $\mathrm{VMoC}$ deviates from the linear relationship at $15 \%$ strain. This is indicative of the so-called yield point, typically observed in ductile materials, which are capable of plastic response at room temperature. As the stress is further increased, rupture/fracture in $\mathrm{VMoC}$ is observed at $20 \%$ strain. In brittle materials, rupture typically occurs suddenly, i.e. without any noticeable change in the stress/strain curves. Furthermore, the electronic structure upon shearing of these materials reveal the same kind of layering as was observed for ductile ternary nitride alloys. The electronic structures of unstrained and tetragonally strained VWC are shown in Figure 5.5 as an example. It is thus clear that the question of ductility in ternary carbide alloys necessitates further investigation. 


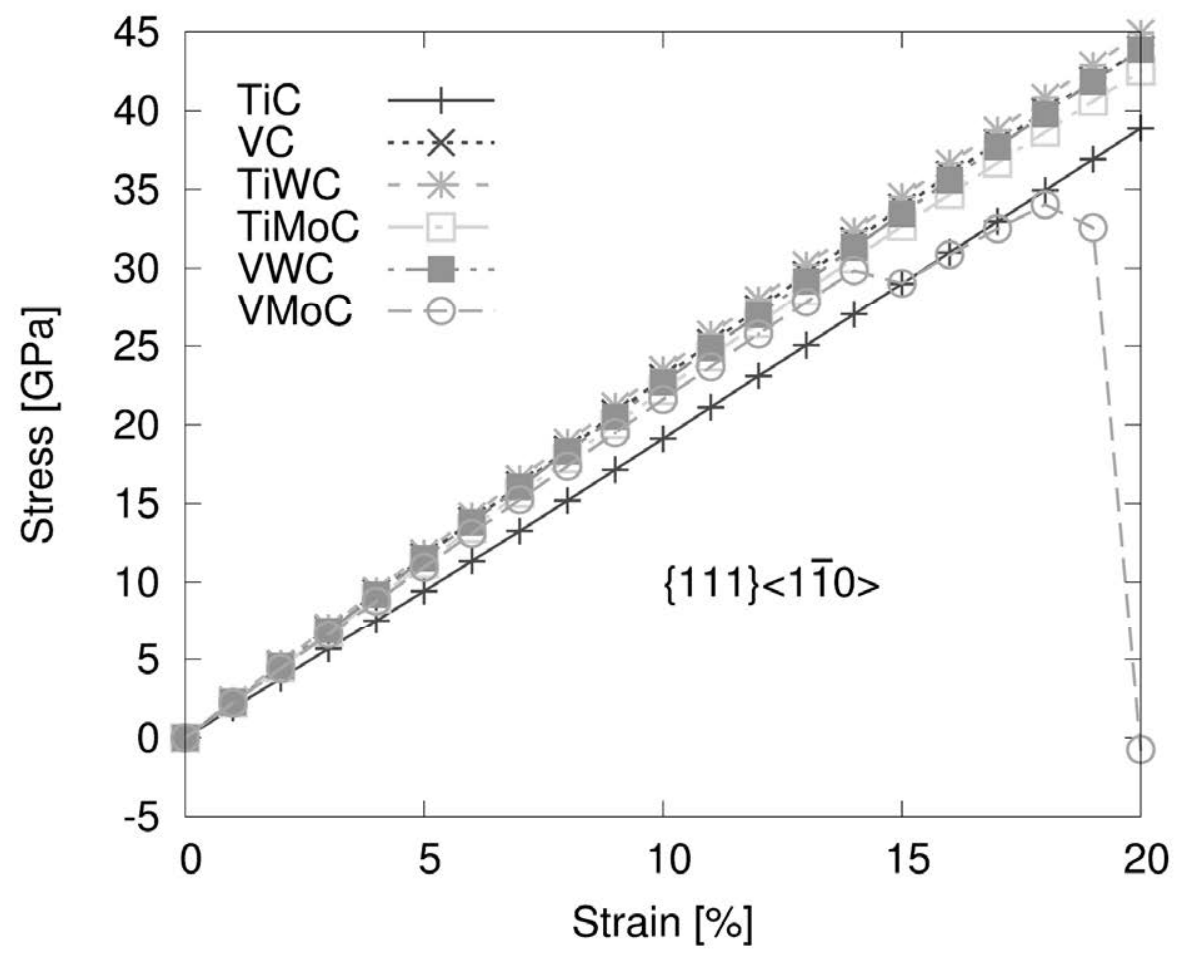

Figure 5.4: Stress/strain curves for $M^{I} M^{2} \mathrm{C}$ alloys along the $\{111\}\langle 1 \overline{1} 0\rangle$ slip system.

(a)

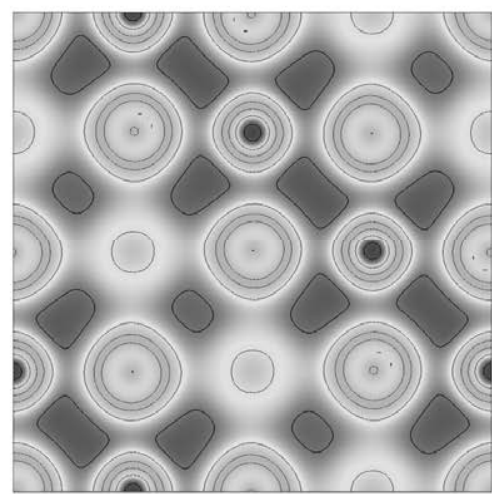

(b)
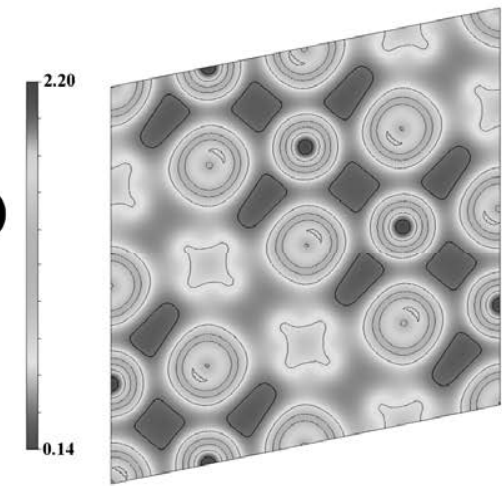

Figure 5.5: Charge densities in the (001) plane of VWC. (a) Unstrained VWC. (b) VWC subject to a tetragonal strain of $10 \%$. 


\section{Contribution to the field and outlook}

Theoretical calculations provide information inaccessible through experimental procedures. Density functional theory allows for the calculation of material properties before synthesis, thereby limiting costly processes to truly promising candidates, while at the same time explaining the origin of said properties via electronic configurations and mechanisms. Classical molecular dynamics, meanwhile, allow for truly large-scale simulations of atomic-scale dynamics, such as diffusion, critical for film growth, and even simulations of entire growth processes.

The main contribution of the work presented herein pertains to the dynamical processes at play during growth of TiN thin films, used as a model system for transition metal nitrides in general. Through the application of classical molecular dynamics, key information about diffusion pathways and decent mechanism of experimentally determined main diffusing species has been uncovered. Of special importance to growth is the result that $\mathrm{Ti}$ adatoms and $\mathrm{TiN}_{2}$ admolecules are the most efficient carriers of $\mathrm{Ti}$ and $\mathrm{N}$ in terms of mass-transport, as they exhibit high diffusivities on both terraces and islands, and descend from islands rapidly. On the other hand, $\mathrm{TiN}_{3}$ admolecules are essentially stationary on both terraces and islands and thus serve as seeds for $3 \mathrm{D}$ growth. To promote 2D film growth, then, the process should ideally be tailored to promote formation of $\mathrm{TiN}_{2}$ molecules and suppress formation of $\mathrm{TiN}_{3}$ molecules. Such effects are critical for understanding the growth mode and microstructural evolution of thin films.

Large-scale growth simulations reveal the effects of altering incident N/Ti flux ratios as well as incident $\mathrm{N}$ kinetic energies, corresponding to $\mathrm{N}_{2}$ partial pressure and substrate bias, respectively, in an experimental setup. At low $\mathrm{N} / \mathrm{Ti}$ flux ratios, local $\mathrm{N}$ deficiency leads to surface roughening via the formation of metal-rich 111 oriented islands. As N/Ti is increased, the formation of these islands is suppressed. At high N/Ti ratios, surface roughening occurs via increased formation of $\mathrm{TiN}_{3}$ admolecules. By increasing the incident $\mathrm{N}$ energy $\mathrm{EN}$, a smaller fraction of $\mathrm{N}$ is retained in the film, and a film closer to ideal layer-by-layer growth can be obtained.

Nevertheless, unanswered questions remain. The simulations carried out here have been limited to the $\operatorname{TiN}(001)$ surface. A natural extension is to consider dynamics of competing surface orientations, such as the (110) and (111) surfaces. Substrate temperature is another key parameter of thin film growth, the effect of which has not been directly studied in this work. The study of temperature is challenging, however, as lower temperatures reduces the rates of all growth-related processes, necessitating longer 
simulation times. With the ongoing increase in computational power available to scientists, such studies should only become more and more feasible in the coming years.

Yet another avenue for future research is to verify the generality of the results obtained for TiN by simulating other transition metal nitrides, such as $\mathrm{VN}$, or even ternary alloys, for example VMoN. To carry out such simulations, a significant effort would first have to be put into the development of interaction potentials for these materials.

The second part of this thesis pertains to the calculation of mechanical properties of materials, chiefly relating to toughness enhancement by increasing ductility. It has been shown using density functional theory that valence electron concentration optimization, via alloying, can be used to create materials with superior ductility, and thereby reduced brittleness. Prior to this work, the predictions were only carried out for highly ordered structures. Here, by applying the Pugh and Pettifor criteria to ordered and disordered structures, it is shown that ductility enhancements for transition metal nitride alloys can be expected regardless of ordering on the metal sublattice. Analysis of the electronic structure reveals that even though disordered alloys do not form perfect layered electronic structures upon shearing, as is the case for ordered alloys, they do form metallic pairs perpendicular to the stress direction which lead to in a similar ductility enhancements. As perfectly ordered structures may be difficult to synthesize, ductility enhancement regardless of atomic ordering is an important finding in this work.

While empirical criteria do not indicate enhanced ductility in cubic $M^{l} M^{2} C$ ternary transition metal carbide alloys, where $M^{l}=\mathrm{Ti}$ or $\mathrm{V}$ and $M^{2}=\mathrm{W}$ or Mo, calculations of stress/strain curves and electronic structure reveal features associated with ductile materials. As such, the predictions are inconclusive and experimental investigations are required. Additionally, expanding the search to include more carbide alloys than the ones studied here may provide further insight into the nature the effect of VEC tuning on ductility.

An avenue for future work is investigating the effects of vacancies in carbide materials, as these materials often are understoichiometric. Additionally, investigating the viability of applying the VEC optimization scheme to other ceramic materials, such as oxides or borides, may be worthwhile for improving performance in these materials. 


\section{References}

[1] S.T. Oyama, The Chemistry of Transition Metal Carbides and Nitrides, Springer Science \& Business Media, 1996.

[2] L.E. Toth, Transition metal carbides and nitrides, Academic Press, 1971.

[3] M. Ohring, Materials science of thin films: deposition and structure, Academic Press, San Diego, CA, 2002. http://www.e-streams.com/es0505/es0505_1873.htm (accessed September 26, 2016).

[4] J.-H. Park, T.S. Sudarshan, Chemical vapor deposition, ASM International, Materials Park, Ohio, 2001.

http://public.eblib.com/choice/publicfullrecord.aspx?p=3002420 (accessed September 26, 2016).

[5] H.S. Nalwa, ed., Handbook of thin film materials, Academic Press, San Diego, 2002.

[6] S. Veprek, M.G.J. Veprek-Heijman, P. Karvankova, J. Prochazka, Different approaches to superhard coatings and nanocomposites, Thin Solid Films. 476 (2005) 1-29. doi:10.1016/j.tsf.2004.10.053.

[7] C.-S. Shin, D. Gall, N. Hellgren, J. Patscheider, I. Petrov, J.E. Greene, Vacancy hardening in single-crystal $\mathrm{TiN}_{\mathrm{x}}(001)$ layers, J. Appl. Phys. 93 (2003) 6025-6028. doi:10.1063/1.1568521.

[8] S.-H. Jhi, J. Ihm, S.G. Louie, M.L. Cohen, Electronic mechanism of hardness enhancement in transition-metal carbonitrides, Nature. 399 (1999) 132-134. doi: $10.1038 / 20148$.

[9] H.W. Hugosson, U. Jansson, B. Johansson, O. Eriksson, Restricting Dislocation Movement in Transition Metal Carbides by Phase Stability Tuning, Science. 293 (2001) 2434-2437. doi:10.1126/science.1060512.

[10] T. Joelsson, L. Hultman, H.W. Hugosson, J.M. Molina-Aldareguia, Phase stability tuning in the $\mathrm{Nb}_{\mathrm{x}} \mathrm{Zr}_{1-\mathrm{x}} \mathrm{N}$ thin-film system for large stacking fault density and enhanced mechanical strength, Appl. Phys. Lett. 86 (2005) 131922. doi:10.1063/1.1884743.

[11] D.G. Sangiovanni, V. Chirita, L. Hultman, Electronic mechanism for toughness enhancement in $\mathrm{Ti}_{\mathrm{x}} \mathrm{M}_{1-\mathrm{x}} \mathrm{N}$ (M=Mo and W), Phys. Rev. B. 81 (2010) 104107. doi:10.1103/PhysRevB.81.104107.

[12] D.G. Sangiovanni, L. Hultman, V. Chirita, Supertoughening in B1 transition metal nitride alloys by increased valence electron concentration, Acta Mater. 59 (2011) 2121-2134. doi:10.1016/j.actamat.2010.12.013.

[13] S. Kodambaka, V. Petrova, A. Vailionis, P. Desjardins, D.G. Cahill, I. Petrov, J.E. Greene, In-situ high-temperature scanning-tunneling-microscopy studies of twodimensional island-decay kinetics on atomically smooth TiN(001), Surf. Rev. Lett. 7 (2000) 589-593. doi:10.1142/S0218625X00000816.

[14] S. Kodambaka, V. Petrova, A. Vailionis, P. Desjardins, D.G. Cahill, I. Petrov, J.E. Greene, TiN(001) and TiN(111) island coarsening kinetics: in-situ scanning tunneling microscopy studies, Thin Solid Films. 392 (2001) 164-168. doi:10.1016/S0040-6090(01)01022-7.

[15] S. Kodambaka, V. Petrova, A. Vailionis, I. Petrov, J.E. Greene, In situ hightemperature scanning tunneling microscopy studies of two-dimensional TiN island coarsening kinetics on TiN(0 0 1), Surf. Sci. 526 (2003) 85-96. doi:10.1016/S00396028(02)02570-0.

[16] F. Watanabe, S. Kodambaka, W. Swiech, J.E. Greene, D.G. Cahill, LEEM study of island decay on $\operatorname{Si}\left(\begin{array}{lll}1 & 1 & 0\end{array}\right)$, Surf. Sci. 572 (2004) 425-432.

doi:10.1016/j.susc.2004.09.014. 
[17] S. Kodambaka, N. Israeli, J. Bareño, W. Święch, K. Ohmori, I. Petrov, J.E. Greene, Low-energy electron microscopy studies of interlayer mass transport kinetics on TiN(1 1 1), Surf. Sci. 560 (2004) 53-62. doi:10.1016/j.susc.2004.05.005.

[18] J. Bareño, S. Kodambaka, S.V. Khare, W. Swiech, V. Petrova, I. Petrov, J.E. Greene, TiN surface dynamics: role of surface and bulk mass transport processes, in: AIP Conf. Proc., AIP Publishing, 2007: pp. 205-224. doi:10.1063/1.2563195.

[19] M.A. Wall, D.G. Cahill, I. Petrov, D. Gall, J.E. Greene, Nucleation kinetics during homoepitaxial growth of TiN(001) by reactive magnetron sputtering, Phys. Rev. B. 70 (2004) 35413. doi:10.1103/PhysRevB.70.035413.

[20] D. Gall, S. Kodambaka, M.A. Wall, I. Petrov, J.E. Greene, Pathways of atomistic processes on TiN(001) and (111) surfaces during film growth: an ab initio study, J. Appl. Phys. 93 (2003) 9086. doi:10.1063/1.1567797.

[21] B. Alling, P. Steneteg, C. Tholander, F. Tasnádi, I. Petrov, J.E. Greene, L. Hultman, Configurational disorder effects on adatom mobilities on Ti1-xAlxN(001) surfaces from first principles, Phys. Rev. B. 85 (2012) 245422. doi:10.1103/PhysRevB.85.245422.

[22] M.A. Wall, D.G. Cahill, I. Petrov, D. Gall, J.E. Greene, Nucleation kinetics versus nitrogen partial pressure during homoepitaxial growth of stoichiometric $\operatorname{TiN}\left(\begin{array}{lll}0 & 0 & 1\end{array}\right)$ : A scanning tunneling microscopy study, Surf. Sci. 581 (2005) L122-127. doi:10.1016/j.susc.2005.03.007.

[23] F.H. Baumann, D. 1. Chopp, T.D. de la Rubia, G.E. Gilmer, J.E. Greene, H. Huang, S. Kodambaka, P. O’Sullivan, I. Petrov, Multiscale Modeling of Thin-Film Deposition: Applications to Si Device Processing, MRS Bull. 26 (2001) 182-189. doi:10.1557/mrs2001.40.

[24] S. Kodambaka, S.V. Khare, V. Petrova, A. Vailionis, I. Petrov, J.E. Greene, Absolute orientation-dependent $\mathrm{TiN}\left(\begin{array}{lll}0 & 0 & 1\end{array}\right)$ step energies from two-dimensional equilibrium island shape and coarsening measurements on epitaxial TiN( $\left.\begin{array}{lll}0 & 0 & 1\end{array}\right)$ layers, Surf. Sci. 513 (2002) 468-474. doi:10.1016/S0039-6028(02)01845-9.

[25] B.W. Karr, I. Petrov, P. Desjardins, D.G. Cahill, J.E. Greene, In situ scanning tunneling microscopy studies of the evolution of surface morphology and microstructure in epitaxial $\mathrm{TiN}(001)$ grown by ultra-high-vacuum reactive magnetron sputtering, Surf. Coat. Technol. 94-95 (1997) 403-408. doi:10.1016/S0257-8972(97)00444-1.

[26] S. Plimpton, Fast Parallel Algorithms for Short-Range Molecular Dynamics, J. Comput. Phys. 117 (1995) 1-19. doi:10.1006/jcph.1995.1039.

[27] M.P. Allen, D.J. Tildesley, Computer Simulation of Liquids, Reprint edition, Oxford University Press, USA, Oxford England; New York, 1989.

[28] D. Frenkel, B. Smit, Understanding Molecular Simulation, Second Edition: From Algorithms to Applications, 2 edition, Academic Press, San Diego, 2001.

[29] R.M. Shroll, D.E. Smith, Molecular dynamics simulations in the grand canonical ensemble: Formulation of a bias potential for umbrella sampling, J. Chem. Phys. 110 (1999) 8295. doi:10.1063/1.478791.

[30] M.I. Baskes, Application of the Embedded-Atom Method to Covalent Materials: A Semiempirical Potential for Silicon, Phys. Rev. Lett. 59 (1987) 2666-2669. doi:10.1103/PhysRevLett.59.2666.

[31] M.S. Daw, M.I. Baskes, Semiempirical, Quantum Mechanical Calculation of Hydrogen Embrittlement in Metals, Phys. Rev. Lett. 50 (1983) 1285-1288. doi:10.1103/PhysRevLett.50.1285.

[32] B.-J. Lee, M.I. Baskes, Second nearest-neighbor modified embedded-atom-method potential, Phys. Rev. B. 62 (2000) 8564-8567. doi:10.1103/PhysRevB.62.8564. 
[33] G. Henkelman, B.P. Uberuaga, H. Jónsson, A climbing image nudged elastic band method for finding saddle points and minimum energy paths, J. Chem. Phys. 113 (2000) 9901-9904. doi:10.1063/1.1329672.

[34] G. Henkelman, H. Jónsson, Improved tangent estimate in the nudged elastic band method for finding minimum energy paths and saddle points, J. Chem. Phys. 113 (2000) 9978-9985. doi:10.1063/1.1323224.

[35] P. Hohenberg, W. Kohn, Inhomogeneous Electron Gas, Phys. Rev. 136 (1964) B864-B871. doi:10.1103/PhysRev.136.B864.

[36] W. Kohn, L.J. Sham, Self-Consistent Equations Including Exchange and Correlation Effects, Phys. Rev. 140 (1965) A1133-A1138. doi:10.1103/PhysRev.140.A1133.

[37] J.P. Perdew, K. Burke, Y. Wang, Generalized gradient approximation for the exchange-correlation hole of a many-electron system, Phys. Rev. B. 54 (1996) 16533-16539. doi:10.1103/PhysRevB.54.16533.

[38] J.P. Perdew, Y. Wang, Accurate and simple analytic representation of the electrongas correlation energy, Phys. Rev. B. 45 (1992) 13244-13249. doi:10.1103/PhysRevB.45.13244.

[39] G. Kresse, J. Hafner, Ab initio molecular dynamics for liquid metals, Phys. Rev. B. 47 (1993) 558-561. doi:10.1103/PhysRevB.47.558.

[40] P.E. Blöchl, Projector augmented-wave method, Phys. Rev. B. 50 (1994) 1795317979. doi:10.1103/PhysRevB.50.17953.

[41] C. Kittel, Introduction to Solid State Physics, Eighth Edition, Wiley, Hoboken, NJ, n.d.

[42] N. Ashcroft, N.D. Mermin, Solid State Physics, 1st ed., Cengage Learning, Boston, MA, n.d.

[43] F. Tasnádi, M. Odén, I.A. Abrikosov, Ab initio elastic tensor of cubic $\operatorname{Ti}_{0.5} \mathrm{Al}_{0.5} \mathrm{~N}$ alloys: Dependence of elastic constants on size and shape of the supercell model and their convergence, Phys. Rev. B. 85 (2012) 144112. doi:10.1103/PhysRevB.85.144112.

[44] M. Moakher, A.N. Norris, The Closest Elastic Tensor of Arbitrary Symmetry to an Elasticity Tensor of Lower Symmetry, J. Elast. 85 (2006) 215-263. doi:10.1007/s10659-006-9082-0.

[45] D.G. Pettifor, Theoretical predictions of structure and related properties of intermetallics, Mater. Sci. Technol. 8 (1992) 345-349.

[46] S.F. Pugh, XCII. Relations between the elastic moduli and the plastic properties of polycrystalline pure metals, Philos. Mag. Ser. 7.45 (1954) 823-843. doi:10.1080/14786440808520496.

[47] R.H.J. Hannink, D.L. Kohlstedt, M.J. Murray, Slip System Determination in Cubic Carbides by Hardness Anisotropy, Proc. R. Soc. Math. Phys. Eng. Sci. 326 (1972) 409-420. doi:10.1098/rspa.1972.0017.

[48] L. Hultman, M. Shinn, P.B. Mirkarimi, S.A. Barnett, Characterization of misfit dislocations in epitaxial (001)-oriented $\mathrm{TiN}, \mathrm{NbN}, \mathrm{VN}$, and $(\mathrm{Ti}, \mathrm{Nb}) \mathrm{N}$ film heterostructures by transmission electron microscopy, J. Cryst. Growth. 135 (1994) 309-317. doi:10.1016/0022-0248(94)90757-9.

[49] P.H. Mayrhofer, D. Music, J.M. Schneider, Influence of the Al distribution on the structure, elastic properties, and phase stability of supersaturated Ti[sub 1-x]Al[sub x]N, J. Appl. Phys. 100 (2006) 94906. doi:10.1063/1.2360778.

[50] A. Zunger, S.-H. Wei, L.G. Ferreira, J.E. Bernard, Special quasirandom structures, Phys. Rev. Lett. 65 (1990) 353-356. doi:10.1103/PhysRevLett.65.353.

[51] J.M. Cowley, An Approximate Theory of Order in Alloys, Phys. Rev. 77 (1950) 669-675. doi:10.1103/PhysRev.77.669. 
[52] A. Anders, A structure zone diagram including plasma-based deposition and ion etching, Thin Solid Films. 518 (2010) 4087-4090. doi:10.1016/j.tsf.2009.10.145.

[53] D.G. Sangiovanni, D. Edström, L. Hultman, I. Petrov, J.E. Greene, V. Chirita, Ab initio and classical molecular dynamics simulations of $\mathrm{N}_{2}$ desorption from $\operatorname{TiN}(001)$ surfaces, Surf. Sci. 624 (2014) 25-31. doi:10.1016/j.susc.2014.01.007.

[54] H. Kindlund, D.G. Sangiovanni, L. Martínez-de-Olcoz, J. Lu, J. Jensen, J. Birch, I. Petrov, J.E. Greene, V. Chirita, L. Hultman, Toughness enhancement in hard ceramic thin films by alloy design, APL Mater. 1 (2013) 42104. doi:doi:10.1063/1.4822440. 


\section{List of Included Publications}

Paper 1 Dynamics of Ti, N, and $\operatorname{TiN}_{\mathrm{x}}(\mathrm{x}=1-3)$ admolecule transport on TiN(001) surfaces

D.G. Sangiovanni, D. Edström, L. Hultman, V. Chirita, I. Petrov, and J.E. Greene

Physical Review B 86, 155443 (2012)

Paper 2 Ti and $\mathrm{N}$ adatom descent pathways to the terrace from atop twodimensional TiN/TiN(001) islands

D. Edström, D.G. Sangiovanni, L. Hultman, V. Chirita, I. Petrov, and J.E. Greene

Thin Solid Films 558, 37-46 (2014)

Paper 3 Effects of atomic ordering on the elastic properties of TiN- and VN-based ternary alloys

D. Edström, D.G. Sangiovanni, L. Hultman, and V. Chirita Thin Solid Films 571, 145-153 (2014)

Paper 4 The dynamics of $\operatorname{TiN}_{\mathrm{x}}(\mathrm{x}=1-3)$ admolecule interlayer and intralayer transport on TiN/TiN(001) islands

D. Edström, D.G. Sangiovanni, L. Hultman, I. Petrov, J.E. Greene, and V. Chirita

Thin Solid Films 589, 133-144 (2015)

Paper 5 Large-scale molecular dynamics simulations of TiN/TiN(001) epitaxial film growth

D. Edström, D.G. Sangiovanni, L. Hultman, I. Petrov, J.E. Greene, and V. Chirita Journal of Vacuum Science \& Technology A 34, 041509 (2016) 
Paper 6 Effects of incident $\mathrm{N}$ atom kinetic energy on TiN/TiN(001) film growth dynamics: a molecular dynamics investigation

D. Edström, D.G. Sangiovanni, L. Hultman, I. Petrov, J.E. Greene, and V. Chirita

Submitted

Paper 7 Elastic properties of TiC- and VC-based ternaries alloyed with MoC and WC

D. Edström, D.G. Sangiovanni, L. Hultman, I. Petrov, J.E. Greene, and V. Chirita

In Manuscript 


\section{Comments on Included Papers}

Copyright of paper 1 belongs to the American Physical Society. Copyright of papers 2, 3, and 4 belongs to Elsevier. Copyright of paper 5 belongs to AVS Publications. Reprints of these papers are included with permission from copyright holders.

1

This paper details CMD studies of the diffusion of Ti and $\mathrm{N}$ adatoms as well as of $\mathrm{TiN}_{\mathrm{x}}$ admolecules, where $\mathrm{x}$ ranges from 1 to 3 , on $\mathrm{TiN}(001)$ infinite terraces at $1000 \mathrm{~K}$. Ti adatoms are revealed to be the most mobile species, followed by $\mathrm{TiN}_{2}$ molecules, whereas $\mathrm{TiN}_{3}$ molecules are essentially stationary and serve as the smallest nucleating cluster.

Contribution of Author: I contributed to the planning of the study, performed part of the calculations and participated in the analysis and discussion of the results and in writing the paper.

\section{2}

This paper details a CMD study of diffusion mechanisms on, and descent mechanisms from, small, square, $\mathrm{TiN} / \mathrm{TiN}(001)$ islands at $1000 \mathrm{~K}$. Ti adatoms are rapidly funneled to island edges, where they descend by means of push-out/exchange. $\mathrm{N}$ adatoms are significantly slower in reaching the island edge and descend via both push-out/exchange and direct hopping.

Contribution of Author: I contributed to the planning of the study, performed part of the calculations, participated in the analysis and discussion of the results, and wrote the paper.

3

This paper details a DFT study of the mechanical properties of transition metal nitride alloys as a function of ordering on the metal sublattice. Ordered structures are shown to have a lower resistance to shear deformation and a higher resistance to tensile deformation than disordered structures. Evaluation of ductility using the Pugh and Pettifor criteria show that all the studied ternary alloys possess superior ductility to the reference binaries, regardless of atomic ordering.

Contribution of Author: I contributed to conceive and plan the study, performed the calculations, participated in the analysis and discussion of the results, and wrote the paper. 
This paper builds on the studies in paper 1 and 2 by studying admolecule transport on TiN/TiN(001) islands. TiN and $\mathrm{TiN}_{2}$ admolecules are shown to have significantly increased migration velocities on islands compared to infinite terraces, whereas $\mathrm{TiN}_{3}$ molecules are essentially stationary and promote multilayer growth.

Contribution of Author: I contributed to conceive and plan the study, performed the calculations, participated in the analysis and discussion of the results, and wrote the paper.

5

This paper details simulations of $\mathrm{TiN} / \mathrm{TiN}(001)$ thin film growth at incident flux ratios of $\mathrm{N} / \mathrm{Ti}=1,2$, and 4 . At $\mathrm{N} / \mathrm{Ti}=1$, films are globally understroichiometric and contain small metal-rich 111-oriented regions. At $\mathrm{N} / \mathrm{Ti}=2$ and 4, films are nearly stoichiometric with excess $\mathrm{N}$ accommodated at $\mathrm{N}$-terminated polar 110 island edges.

Contribution of Author: I contributed to conceive and plan the study, performed the calculations, participated in the analysis and discussion of the results, and wrote the paper.

\section{6}

This paper details simulations of $\mathrm{TiN} / \mathrm{TiN}(001)$ thin film growth at incident $\mathrm{N}$ kinetic energies of $\mathrm{E}_{\mathrm{N}}=2$ and $10 \mathrm{eV}$. At $\mathrm{E}_{\mathrm{N}}=2 \mathrm{eV}$, grown films are globally overstoichiometric with islands bounded by $\mathrm{N}$-terminated 110 edges. $\mathrm{E}_{\mathrm{N}}=10 \mathrm{eV}$ results in smoother films with compositions closer to stoichiometric. Local N-deficiency leads to formation of 111oriented regions.

Contribution of Author: I contributed to conceive and plan the study, performed the calculations, participated in the analysis and discussion of the results, and wrote the paper.

\section{7}

This paper details a DFT study of the mechanical properties of ternary transition metal carbide alloys. According to the Pugh and Pettifor criteria, none of the studied alloys are predictied to be ductile. However, calculations of stress/strain curves and charge densities reveal features typically associated with ductile materials, motivating further study of these materials.

Contribution of Author: I contributed to conceive and plan the study, performed the calculations, participated in the analysis and discussion of the results, and wrote the paper. 


\section{Included Papers}

The articles associated with this thesis have been removed for copyright reasons. For more details about these see:

http://urn.kb.se/resolve?urn=urn:nbn:se:liu:diva-132272 